\title{
Le sanctuaire de la Combe de l'Ermitage à Collias (Gard)
}

\author{
Michel Christol, Jean-Luc Fiches, Dominique RabAY
}

\begin{abstract}
Résumé: Le nouvel examen d'un dossier épigraphique connu depuis longtemps et la recherche sur place de remplois nombreux dans une église médiévale permettent de mieux caractériser ce sanctuaire rural situé à moins de $20 \mathrm{~km}$ de Nîmes. En rapport avec une source, il est fréquenté dès le $\mathrm{I}^{\mathrm{er}}$ s. av. J.-C.; doté ensuite d'un portique, il reste actif jusqu'au $\mathrm{II}^{\mathrm{e}}$, voire au $\mathrm{III}^{\mathrm{e}} \mathrm{s}$. On y honore alors, aux côtés de Jupiter qui y joue un rôle fédérateur, plusieurs divinités indigènes dont certaines sont associées à des localités voisines.
\end{abstract}

Mots clefs: Aramo (divinité), autels votifs et funéraires, Budenicenses, Coriobedenses, Jupiter, Mars Budenicus, Matres, Minerve, pilier votif, portique, roue, source, Sulevia [L]edennica (divinité), supports d'offrandes.

Abstract: The new examination of some well known epigraphic documents and the search for numerous re-employed fragments in a medieval church, on site, have set up a better characterization of this rural sanctuary which is located at less than $20 \mathrm{~km}$ from Nîmes. Being linked to a source, it was used as soon as the $1^{\text {st }} \mathrm{c}$. BC.; later equipped with a portico, and it remained in activity until the $2^{\text {nd }} \mathrm{c}$. or even the $3^{\text {rd }} \mathrm{c}$. Several indigenous divinities were worshipped here, besides Jupiter who plays a unifying role; some of them are associated with neighbouring localities.

Key words : Aramo (divinity), votive and funerary altars, Budenicenses, Coriobedenses, Jupiter, Mars Budenicus, Matres, Minerve, votive pillar, portico, wheel, source, Sulevia [L]edennica (divinity), supports for offerings.

\section{LE SITE ET SA SITUATION}

Dans la partie calcaire urgonienne de la garrigue de Nîmes, la Combe de l'Ermitage est un des nombreux vallons qui mènent un ruisseau temporaire à caractère torrentiel vers le Gardon (Marcelin 1926, 127-140; Fabre 1980, 4852). Suivant un axe rectiligne sud-nord, c'est un de ceux qui s'enfoncent le plus loin dans le massif, jusqu'à $1 \mathrm{~km}$ au nord du village de Cabrières. À l'amont, il est caractérisé par des rideaux de culture particulièrement développés dans des limons épais. Il prend ensuite la forme d'une petite vallée à fond plat, à pente faible et aux versants rocheux convexes jusqu'au Mas de Laval qui se situe à peu près au milieu de sa longueur (fig. 1). Â partir de ce point, le vallon se transforme en un petit canyon envahi par une végétation inextricable et dont le talweg présente une pente prononcée et heurtée (fig.2). C'est dans ce canyon que se trouve la source karstique de l'Ermitage de Notre-Dame-de-Laval, dominée par un petit ressaut de cascade (Fabre, Pey 1997, 128-130). En aval de la source, le canyon, aux versants escarpés et dans lequel on a reconnu cinq grottes ou abris occupés durant la Préhistoire, a une pente plus faible qui facilite l'accès à la source, depuis la rive droite du Gardon qui coule $3 \mathrm{~km}$ plus bas.
Depuis Nîmes, au sud-ouest, routes, chemins puis sentiers permettent d'atteindre le site de Notre-Dame-deLaval après un parcours de $18 \mathrm{~km}$ environ (fig. 3). C'est un «cirque» naturel bordé de falaises calcaires et boisé de chênes verts, dominé par le petit oppidum du Paradas de Clastre qui semble avoir été occupé entre le $\mathrm{VI}^{\mathrm{e}}$ et le $\mathrm{III}^{\mathrm{e}}$ s. av. J.-C. $\left(C A G, 30-2,330\right.$, notice $\left.085, \mathrm{n}^{\circ} 5^{*}\right)$. Située

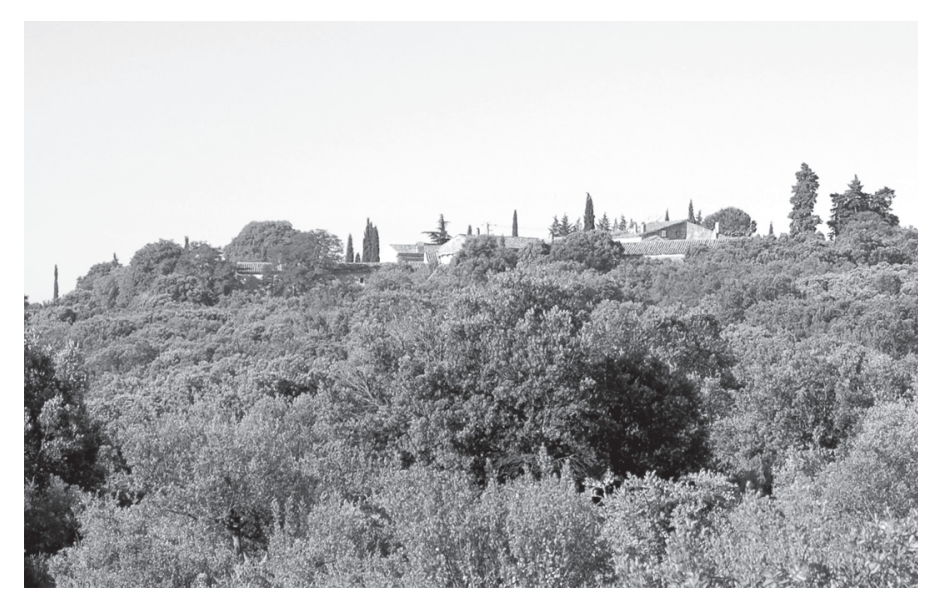

Fig. I : Le Mas de Laval vu du nord (cliché J.-L. Fiches). 
au pied sud-est de cette reculée, la source comporte aujourd'hui un petit bassin de rétention (fig. 4). Toujours au pied des falaises, à $80 \mathrm{~m}$ vers le nord-ouest, s'élève, près d'un ermitage, une chapelle (fig. 5) qui domine des terrasses en pierre sèche entre lesquelles un escalier donne accès aux bâtiments depuis le ruisseau enjambé par un petit pont en pierre à une arche. Cet accès et les terrasses ont fait l'objet de travaux de restauration dans le cadre d'un chantier de réinsertion, à la suite de la crue de septembre 2002 qui avait fortement endommagé le chemin depuis Collias.

Classés Monument historique en 1931, les bâtiments sont implantés au-dessus d'une terrasse où s'élève un autel en plein air: sur une plate-forme intermédiaire (fig. 6), se trouvent l'église actuellement en service et une chapelle en ruine, autrefois entourée d'un cimetière; au-dessus un abri sous roche auquel on accède par un escalier devant l'église, a accueilli l'habitat troglodytique de l'ermite.

L'abbé Bayol, qui nomme cet abri la Grotte des Ermites, a effectué des fouilles sur la terrasse intermédiaire, devant l'église. Il y a reconnu des occupations du Paléolithique (silex, faune, crâne humain à $5 \mathrm{~m}$ de profondeur) et du Néolithique (outillages lithiques et osseux, meules dormantes, broyeurs, percuteurs, etc.) ainsi que des objets divers qu'il attribue aux âges des métaux (Bayol 1980, 11). Recherchant aussi l'emplacement du cimetière médiéval, il a trouvé par ailleurs, à 2,10 m de profondeur et sur le sol vierge, des squelettes alignés vers le levant dont l'un portait une boucle de ceinture en bronze et une coquille au niveau de l'épaule. Ces tombes étaient scellées, à $0,80 \mathrm{~m}$ de la surface, par une couche contenant des os humains pêle-mêle et un squelette complet face contre terre (Bayol 1980, 19). Enfin, à l'occasion d'une fouille limitée, l'abbé a découvert, un peu en dehors de l'église ruinée, «deux sépultures jumelles» qui contenaient «des tessons de poteries noires, fines et lustrées» dans lesquels il a vu des productions grecques, mais les tombes sont vraisemblablement médiévales (Bayol 1980, 14).

\section{Les édifices ReligieuX DU Moyen ÂGE}

Pour G. Durand (Durand 1992), qui rappelle que l'ermitage et le vocable Notre-Dame n'apparaissent qu'au $\mathrm{XVII}{ }^{\mathrm{e}} \mathrm{s}$. alors que la communauté de Laval a vraisemblablement disparu au $\mathrm{XV}^{\mathrm{e}} \mathrm{s}$., les deux édifices religieux correspondaient, au Moyen Âge, à l'église paroissiale Saint-Étienne et à l'église funéraire de Laval. On ne peut exclure cependant que le village et son cimetière aient été implantés plus haut sur le plateau, dans le secteur plus propice aux cultures où s'élève aujourd'hui le Mas de Laval dont les bâtiments couvrent près d'un hectare. En effet, c'est dans une terre cultivée, à 3 ou $400 \mathrm{~m}$ au sud du Mas qu'ont été observées, dans le talus d'un chemin creux, des inhumations en dalles $\left(\mathrm{VI}^{\mathrm{e}}-\mathrm{XII}{ }^{\mathrm{e}} \mathrm{s}\right.$.) enfouies à

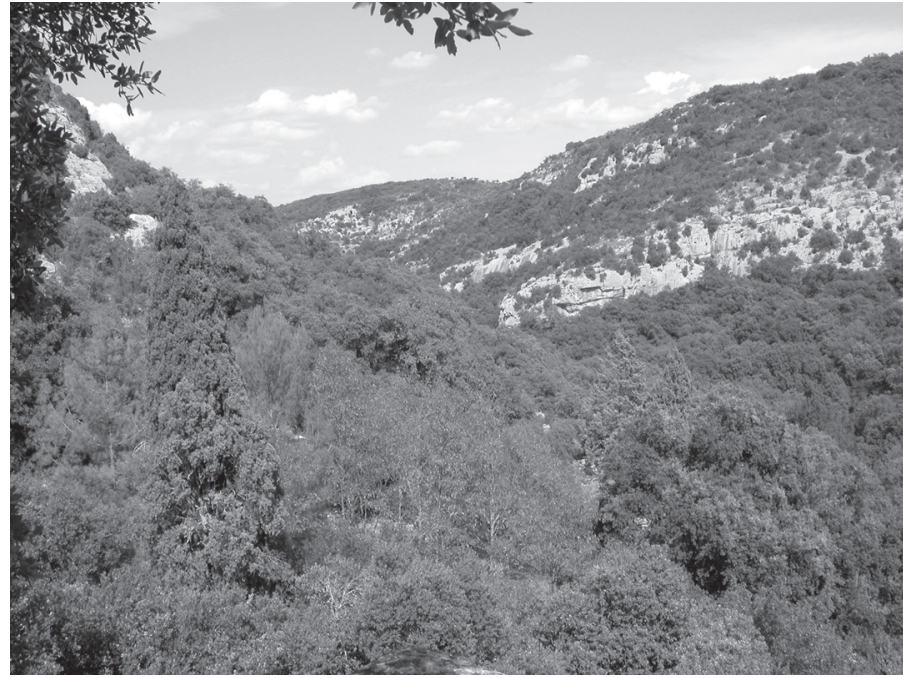

Fig. 2: La Combe de l'Ermitage telle qu'elle apparaît au-dessus du site lorsqu'on vient du sud (cliché J.-L. Fiches).

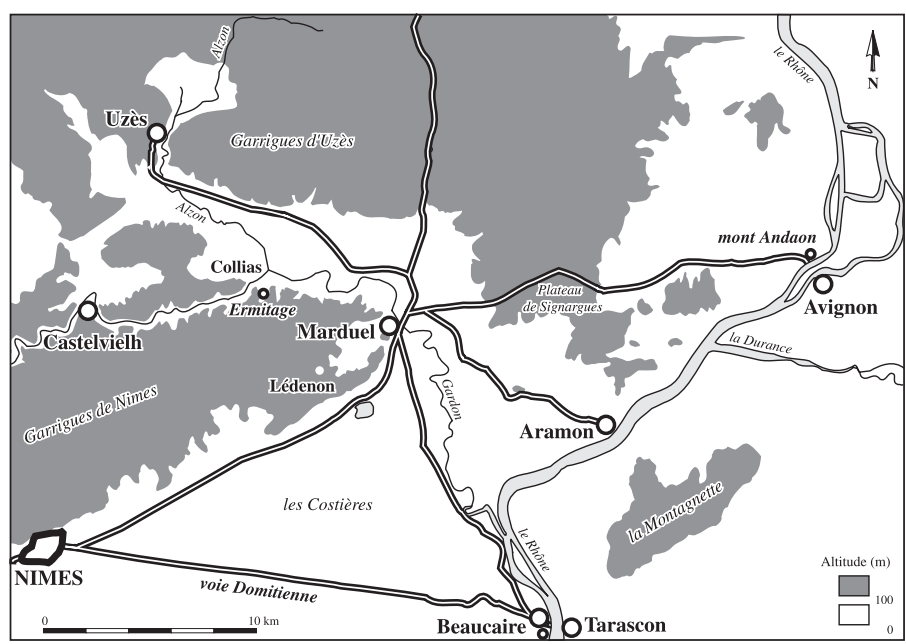

Fig. 3 : Situation de l'Ermitage entre Nîmes, Uzès et Avignon: agglomérations (grands cercles), sanctuaires ruraux (petits cercles) et voies antiques (DAO Th. Panouillères).

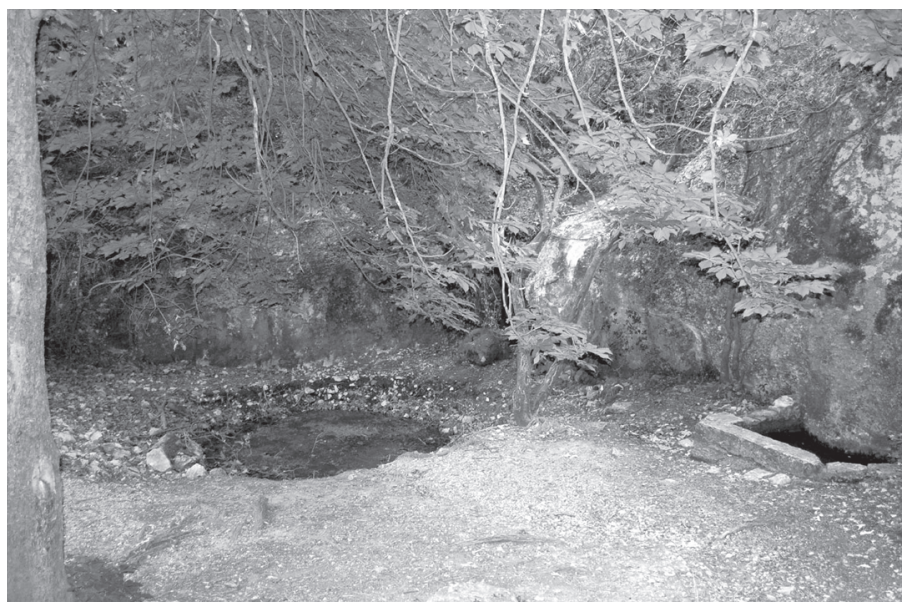

Fig. 4: La source de la Combe de l'Ermitage (cliché J.-L. Fiches). 


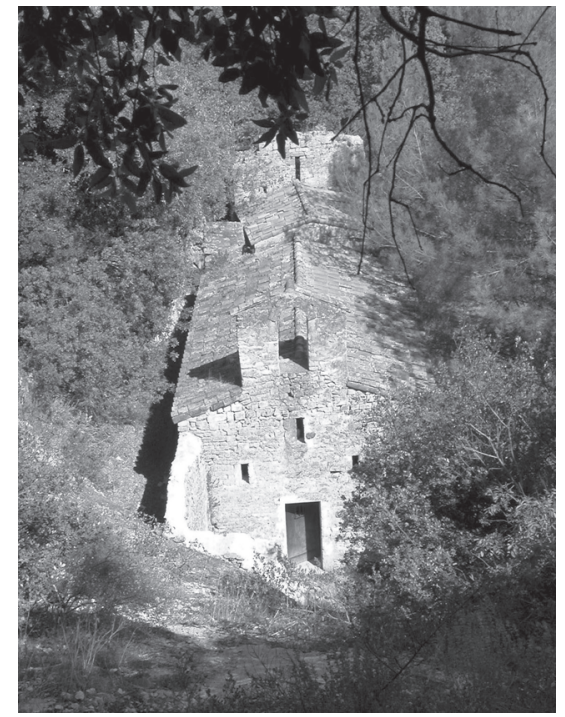

Fig. 5 : L'église de Notre-Dame-deLaval depuis l'escarpement qui borde le site (cliché J.-L. Fiches).

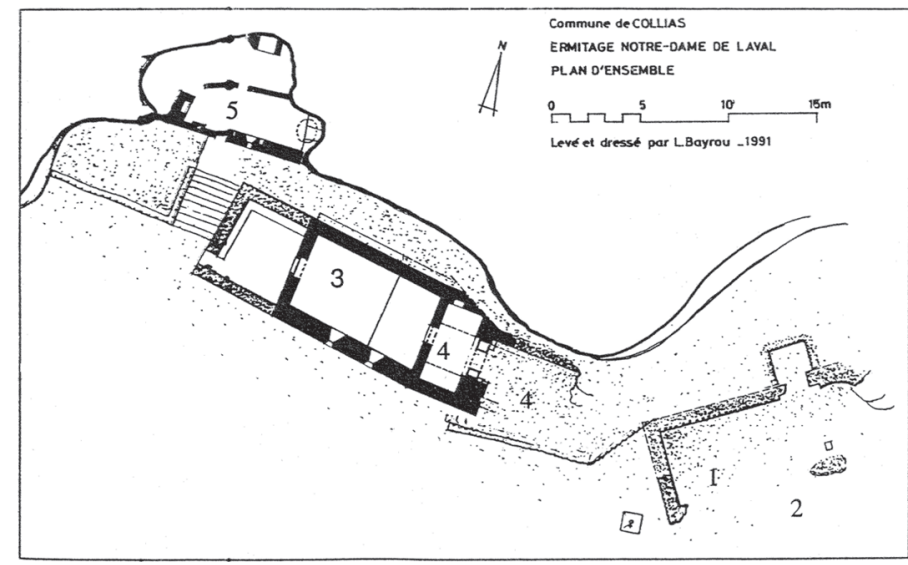

Fig. 6: Notre-Dame-en-Laval. Église ruinée à abside outrepassée (I), cimetière médiéval (2), partie de la nef romane encore en service (3), travée de plan barlong et chevet surélevés (4), ermitage (5).

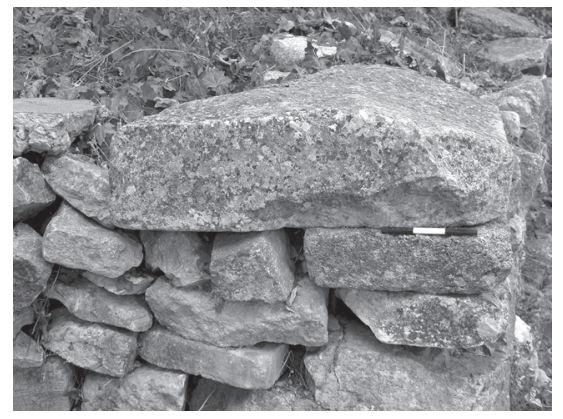

Fig. 7 : Couvercle de sarcophage en remploi sur un muret en bordure de l'accès à l'église et à l'ermitage (cliché J.-L. Fiches). près de $1 \mathrm{~m}$ de profondeur (Mazauric 1905) et non à l'ermitage (mention erronée de $C A G, 30-2,333$, notice 085 , $\mathrm{n}^{\circ} 11^{*}$, qui précise de façon abusive: tombes des $\mathrm{III}^{\mathrm{e}}-\mathrm{IV}^{\mathrm{e}} \mathrm{s}$.).

Caractérisée par une nef unique et une abside outrepassée à l'intérieur, quadrangulaire ou plus vraisemblablement semi-circulaire à l'extérieur, l'église ruinée serait, pour G. Durand, antérieure à l'an mil. Cependant, son hypothèse, selon laquelle un martyrium serait à l'origine du culte chrétien, demeure fragile. Certes, à l'occasion d'une visite sur le site en août $2005^{1}$, on a observé la présence, sur un muret qui borde l'accès à l'autre église, d'un fragment de couvercle de sarcophage (fig.7), mais celui-ci n'est pas caractéristique de la série bien connue dans la région à partir du $\mathrm{VI}^{\mathrm{e}} \mathrm{s}$; ; il pourrait, en fait, ne pas être antérieur aux $\mathrm{XI}^{\mathrm{e}}-\mathrm{XII}^{\mathrm{e}} \mathrm{s}$.

L'église actuellement en service, au nord-ouest de la précédente, remonterait au $\mathrm{XI}^{\mathrm{e}}$ siècle mais elle a été plusieurs fois remaniée, en particulier à la fin du $\mathrm{XVII}^{e} \mathrm{~s}$. où la nef romane a été raccourcie, couverte d'un berceau en plein-cintre et fermée par un nouveau mur transversal percé d'une porte et de trois petites baies sous un clocherarcade (fig. 8). La nef romane est bâtie en moellons de petit appareil allongés ou cubiques parmi lesquels on a observé, en 2005, un remploi de concrétions provenant de l'aqueduc de Nîmes (fig.9) dans la partie inférieure de la façade sud (Paillet 2000). Celle-ci est percée de deux larges portes en plein cintre légèrement surhaussé et comporte, dans sa partie haute, «une rangée de corbeaux en pierre, à encoche, de forme arrondie, destinés à porter des sablières » (Durand 1992, 139).

La nef communique, par une porte étroite en plein cintre, avec une partie surélevée qui est désaxée. Cette travée de plan barlong, qui constitue un transept bas, ouvre sur un espace plus étroit, aujourd'hui ruiné (fig. $6, \mathrm{n}^{\circ} 4$ ); ce chevet, qui daterait du $\mathrm{XII}^{\mathrm{e}} \mathrm{s}$., a pu être fortifié et recevoir un clocher-tour.

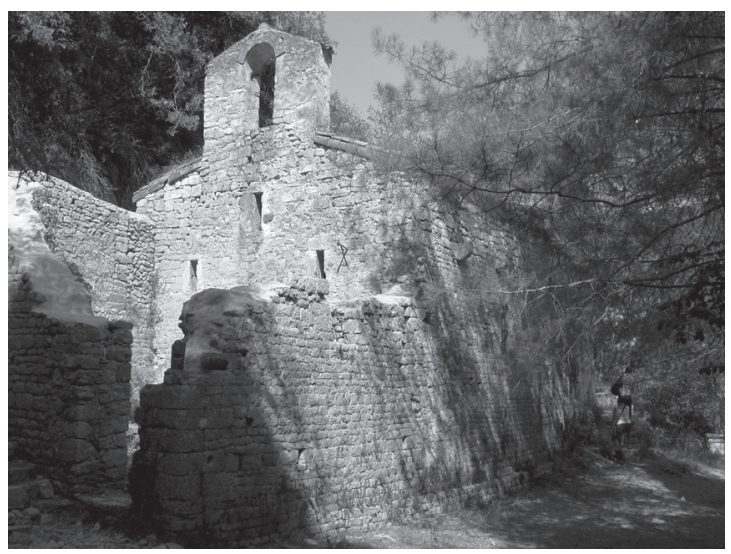

Fig. 8: La nef romane raccourcie à la fin du XVII es. par un mur transversal, surmonté d'un clocher-arcade (cliché J.-L. Fiches). 
À n'en pas douter, le plan inhabituel de cet édifice a dû tenir compte d'une construction antérieure dont la fondation, bien visible à l'extérieur du côté sud, est faite de blocs cyclopéens qui supportent la travée de plan barlong et contre lesquels s'appuient les moellons de la nef romane (fig. 10). Ces blocs, grossièrement alignés, ont d'ailleurs une orientation légèrement divergente par rapport à celle des constructions médiévales. Une origine romaine est tout à fait envisageable pour cet aménagement en raison de la présence de tegulae alentour ${ }^{2}$ et surtout de blocs inscrits en remploi dans les bâtiments médiévaux et modernes ${ }^{3}$.

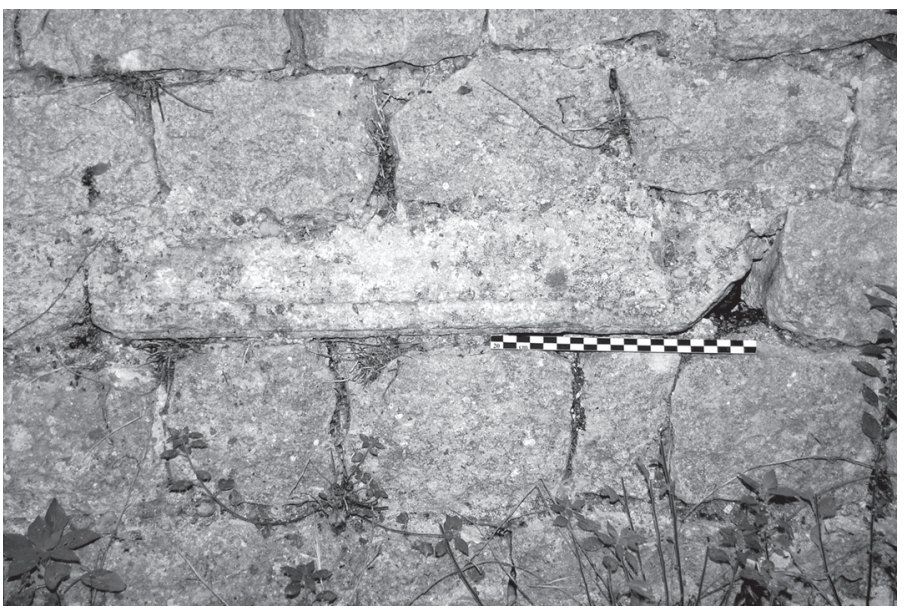

Fig. 9: Remploi de concrétions provenant de l'aqueduc de Nîmes (paroi interne) dans la nef romane (cliché J.-L. Fiches).

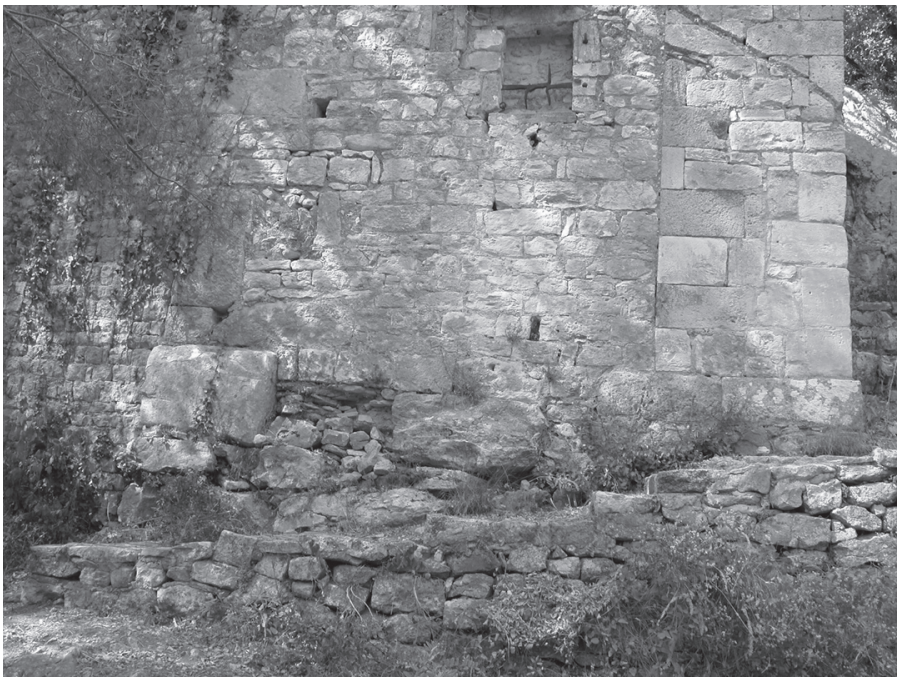

Fig. 10: Substruction de gros blocs sur laquelle s'appuie le mur en petit appareil de la nef romane (à gauche) et repose la travée de plan barlong ainsi que le chevet (à droite) (cliché J.-L. Fiches).

\section{UNE AGGLOMÉRATION GALLO-ROMAINE?}

Substructions et monuments votifs non loin d'une source sont donc les indices qui, depuis longtemps, ont fait penser que le site de l'Ermitage avait été occupé, dans l'Antiquité, par un sanctuaire. Mais on doit également mentionner la présence d'autels funéraires $(C A G, 30-2$, 332-333, notice $\left.085, \mathrm{n}^{\circ} 10^{*}\right)$ : les épitaphes de Lucius Antonius Macrinus (CIL, XII, 2975; IAN, 550; HGL, XV, 1513), d'une femme (CIL, XII, 2998; HGL, XV, 1519; IAN, 552), de M. Attius Secundus (CIL, XII, 2978; HGL, XV, 1517; IAN, 551; ILGN, 400; Espérandieu 1907-1938, IX, 153, n 6815), d'Appius Annius Nicephorus (CAG, 30-2, 333) et de Q. Cosconius Firminus (CIL, XII, 2976; HGL, XV, 1514; ILGN, 401; Espérandieu 1907-1938, X, $\left.229, n^{\circ} 7628\right)$. Cette dernière, considérée comme perdue par la $C A G$ (fig. 11), se trouve certainement dans ce qu'il est convenu d'appeler le musée lapidaire sur le site, posée à plat, son inscription contre le sol.

Cette série est complétée par deux fragments dont l'un est perdu et l'autre conservé à la mairie de Collias (HGL, 1520; CAG, 30-2, 333) et par un bloc en remploi de la porte intérieure dont les dimensions $(55 \times 70$, h. $112 \mathrm{~cm})$ correspondent à un autel funéraire.

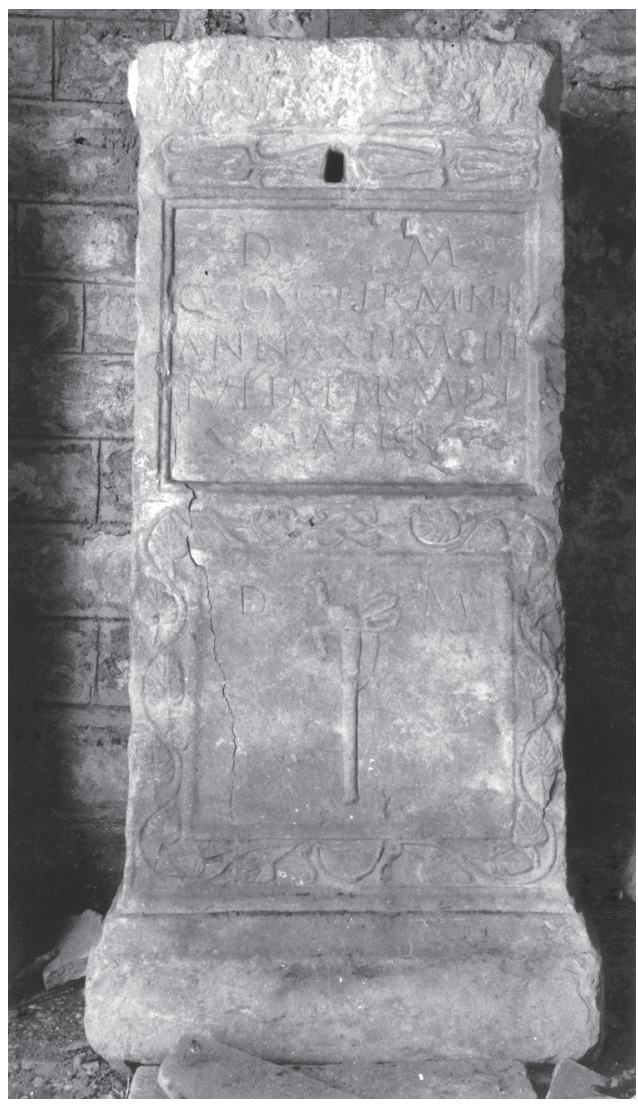

Fig. I I :Autel funéraire de Q. Cosconius Firminus (cliché V. Lassalle, Musée de Nîmes) 
Les inscriptions les mieux conservées se placent dans une période comprise entre la fin du I ${ }^{\mathrm{er}}$ et le début du $\mathrm{III}^{\mathrm{e}} \mathrm{s}$. et se rapportent à des familles différentes de citoyens romains dont l'un, L. Sabinius Severus, l'est de fraîche date. Ils appartiennent tous à des autels dont plusieurs sont ornés de rinceaux ${ }^{4}$, ce qui laisse supposer un recrutement parmi des couches aisées de la population de la cité. La présence de ces monuments funéraires a pu laisser penser que le sanctuaire était associé à une petite agglomération sur le site même (CAG, 30-2, 331, notice 085, titre III), mais, comme on l'a déjà indiqué pour l'habitat médiéval, il est possible que l'établissement gallo-romain soit à rechercher dans le secteur du Mas de Laval, beaucoup plus favorable, en dehors du point d'eau, à une implantation humaine d'une certaine importance.

\section{LA SOURCE À L'ORIGINE DU SANCTUAIRE}

Trouvé avant 1869 , dans le mur sud de la chapelle de l'Ermitage et transporté vers 1884-1888 au musée de Nîmes, le document votif le plus ancien est un pilier de calcaire dur, de section presque carrée $(23 \times 20-24 \mathrm{~cm})$, haut de $1,29 \mathrm{~m}$, fruste à la base sur $33 \mathrm{~cm}$ (partie destinée à être enterrée) et dont le sommet a été peut-être retaillé. En raison de son inscription gallo-grecque, il ne peut guère être postérieur au milieu du I ${ }^{\mathrm{er}} \mathrm{s}$. av. J.-C. (fig. 12). Il porte, sur neuf lignes, une dédicace, par Ekilios, fils de Rumos (ou Riumos), aux Déesses-Mères, qualifiées par l'épithète andoounnabo:

$\varepsilon \kappa \imath \lambda \iota / \sigma \rho[?] \circ v \mu \alpha v[\imath] o \sigma \alpha v / \delta o o v v / v \alpha \beta o \quad \delta /[?] \delta \beta \rho \alpha \tau o /[v]$ $\delta \varepsilon \kappa \alpha v / \tau \varepsilon v$ : CIL, XII, 5887; Lejeune 1985, 250-254 (G183), fig. 218-219; $C A G, 30-2,331$, notice $085, n^{\circ} 8^{*}$.
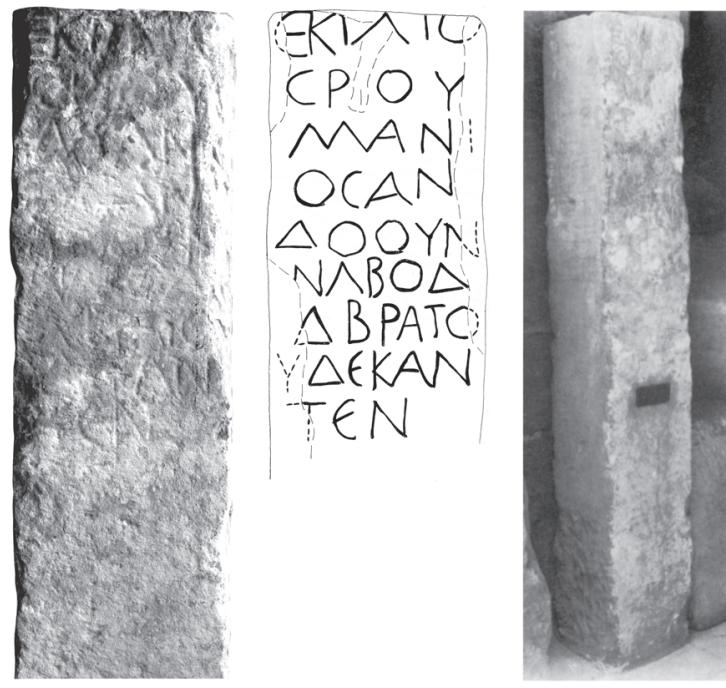

Fig. 12: Pilier portant une dédicace gallo-grecque aux Déesses-Mères par Ekilios, fils de Rumos (ou Riumos) (d'après Lejeune 1985).
La désignation des Mères a fait l'objet d'hypothèses divergentes. Traditionnellement, on leur conférait une valeur topique, en rapport avec le Mont Andaon de Villeneuve-lès-Avignon ${ }^{5}$. Mais si l'on suit l'interprétation de P.-Y. Lambert, reprise par X. Delamarre, ce seraient «les divinités de la source d'en bas» (Delamarre 2001, 42, sv. Andounna). Cette qualification topographique par rapport au plateau du Mas de Laval, situé au sud, pourrait ainsi désigner quel était alors l'accès principal au sanctuaire alors qu'aujourd'hui le rattachement administratif du lieu à la commune de Collias met en valeur le cheminement depuis le Gardon.

Un pilier du même type, dédié à une divinité masculine, a été trouvé à Nîmes même (Lejeune 1985, 284-287, G206). L'invocation de ces déesses connues en collectivité, surtout présentes en Gaule (Duval 1993, 55-56), atteste sans doute que la source de l'Ermitage était l'objet d'un culte comme la Fontaine de Nîmes où celui des Mères nîmoises est connu par une inscription gallo-grecque (Lejeune 1985, 282-284, G-205). Le culte des Mères est également attesté à Glanum par deux inscriptions gallo-grecques (Lejeune 1985, G-64 et 65), l'une aux Mères glaniques, l'autre avec épiclèse роклоıбıаßo («aux écoutantes»?) à mettre en parallèle avec l'appellation de Collias.

\section{LA PRÉSENCE D'UN BÂTIMENT}

Le site a également livré une plaque, qui devait être apposée sur une construction, vraisemblablement dans un emplacement remarquable, car on pouvait souhaiter mettre en valeur un tel acte votif. C'est dans la petite cour qui précède immédiatement la chapelle qu'elle se trouvait, d'après les mots de G. Charvet. Il ne s'agit nullement d'une situation d'origine: la plaque a sans aucun doute fait l'objet de transferts et de manipulations avant même d'être récupérée et transportée au Musée archéologique de Nîmes. Elle est de forme rectangulaire (h. 46; 1. 58; ép. $7,5 \mathrm{~cm}$ ), et l'inscription se trouve dans un cadre mouluré (le champ épigraphique mesure 37 x 49,5 cm). Dans son état actuel, elle présente d'assez nombreuses dégradations, mais on peut néanmoins procéder aux indispensables relectures ou révisions (fig. 13). Il s'agit d'une dédicace au dieu Aramo, dont c'est pour l'instant la seule attestation épigraphique (CIL, XII, 2971 et p. 832; HGL, XV, 1510; $I A N, 548$; voir $C A G, 30-2,332$, notice $085, \mathrm{n}^{\circ} 9^{*}$ ). La mise en page du texte est très dégagée et la gravure est de bonne facture. On peut envisager de dater le texte de la seconde moitié du I ${ }^{\mathrm{er}} \mathrm{s}$. ap. J.-C.

\section{Aramoni}

porticum

Licinia P(ubli) f(ilia) / Acceptilla

ex voto d(e) s(ua) p(ecunia) fecit.

«Au dieu Aramon, Licinia Acceptilla, fille de Publius, à la suite de son vœu a offert de ses propres deniers ce portique». 
Le texte précise que c'est à la suite d'un vœu que Licinia Acceptilla a offert de ses propres deniers le portique qui devait supporter, à un emplacement choisi, la plaque inscrite. Il s'agit d'une offrande significative d'un embellissement $^{6}$, et le formulaire vise à mettre en valeur l'engagement financier de la personne. À tout le moins il permet de situer Licinia Acceptilla au sein de la catégorie des généreux donateurs du sanctuaire, même si le caractère contraignant de l'acte est évident (ex voto).

Les traits distinctifs de cette personne peuvent se déduire aussi de sa dénomination. Le cognomen Acceptilla, dont on ne saurait discuter la forme latine, présente toutefois des signes linguistiques locaux ${ }^{7}$, qui permettent de rattacher cette femme aux familles d'origine indigène qui gagnèrent assez rapidement la citoyenneté romaine et constituèrent l'élite socio-politique de la grande cité de Nîmes. D'abord il s'agit du suffixe -illus/-illa ${ }^{8}$, bien attesté aussi dans l'anthroponymie celtique parmi les noms de forme hypocoristique ${ }^{9}$ et maintenu dans les usages que l'on relève dans la cité de Nîmes: ainsi, on connaît à Lattes Divecillus (Divecillus Carionis f(ilius): $A E, 1972,327$ ) ; à Cournonsec Vossillus (CIL, XII, $4206=$ HGL, XV, 1895, cf. Gallia, 31, 1973, 491); à Nîmes Adgubillus (CIL, XII, 2042), Atepilla (Atepilla Atessatis f(ilia): CIL, XII, 3429), etc. Ensuite il s'agit du cognomen Acceptus, qui correspond à un participe passé latin ${ }^{10}$, mais qui, dans l'anthroponymie de cette colonie de droit latin, a été remarquablement utilisé. Il apparaît dès lors dans la dénomination des couches sociales les plus diverses. C'est ainsi qu'à Narbonne on relève même Accepta comme cognomen d'une affranchie, Fadia T(iti) l(iberta) Accepta (CIL, XII, 4810). On insistera surtout sur les inscriptions en provenance de cette cité. Il y a d'autres exemples en Narbonnaise: CIL, XII, 1384 à Vaison (peut-être un artisan de bon niveau, opifex lapidarius), CIL, XII, 1964 à Vienne

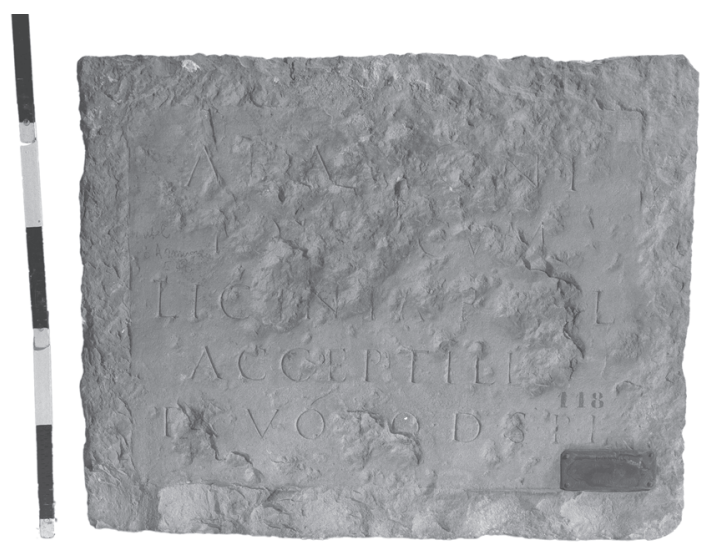

Fig. 13: Dédicace au dieu Aramon, par Licinia Acceptilla qui a offert de ses propres deniers le portique qui devait supporter, à un emplacement choisi, la plaque inscrite (cliché Foliot-Réveillac, Centre Camille-Jullian). (deux attestations), et surtout CIL, XII, 852 à Arles (Accepta Servaci f[ilia], épouse de Q[uintus] Melius Toutonis f[ilius] Flavos) pour un personnage que la tribu Voltinia rattache incontestablement à une cité latine, peutêtre Nîmes.

Dans une inscription de Pouzilhac (partie rhodanienne de la colonie de Nîmes), Accepta est l'épouse pérégrine d'un citoyen romain, Sex(tus) Valerius Auctus (CIL, XII, $2804=H G L, X V, 1525)$; à Nîmes, Acceptus Escingomari f(ilius) et Vernio Accepti f(ilius) sont aussi de condition pérégrine et, qui plus est, leur dénomination est marquée par l'anthroponymie celtique ${ }^{11}$; à Cabrières, dans le même contexte social et juridique, on connaît Acceptus T(iti) f(ilius) (Gallia, 37, 1979, $537=I A C N, 14$ d'où $A E$, 1995, 1070). Enfin, dans un niveau social plus élevé, comparable à celui dans lequel se mouvait vraisemblablement Licinia Acceptilla, on connaît [-] Aemilius L(ucii) f(ilius) Volt. Acceptus, quattuorvir et pontife, c'est-à-dire notable du meilleur rang (Bauquier 1946, 2).

Sans que l'on puisse pour l'instant placer Licinia Acceptilla et sa famille au sein de l'élite la plus relevée, on estimera néanmoins que ce vœu paraît marquant d'une certaine aisance. On a surtout des témoignages sur les Licinii nîmois par des inscriptions de sévirs augustaux (Christol et al. 1987, 388-398, d'où $A E, 1987,752$ ). Un seul exemple de magistrat est connu: encore s'agit-il d'un questeur, c'est-à-dire d'une personne appartenant aux niveaux inférieurs de l'élite politique ${ }^{12}$. Une flaminique apparaît aussi, Licinia L(ucii) f(ilia) Flavilla, mais il n'est pas impossible que l'obtention de cette fonction prestigieuse ne résulte du rang de son mari, Sex(tus) Adgennius Macrinus, membre de l'ordre équestre (CIL, XII, $3175=$ $H G L, \mathrm{XV}, 214)$. Il n'est donc pas inutile de donner tout son poids à ce document votif, si l'on veut apprécier le profil sociologique de la donatrice.

L'ancrage local du rayonnement de cette personne mérite aussi réflexion. L'enracinement de son don à Collias montre, de lui-même, l'importance qu'avait le lieu, dans le territoire de Nîmes. Il faut aussi relever que le nom du dieu gallo-romain Aramo ne peut que renvoyer à la localité qui actuellement porte le nom d'Aramon (Genty et al. 2002). Elle est distante de Collias de près d'une vingtaine de kilomètres. Aussi il est difficile de considérer qu'Aramo soit à Collias le dieu principal ou une divinité d'origine. La construction de ce portique constitue non un transfert du dieu, mais un déplacement du culte, ou un renforcement de son extension ${ }^{13}$. Elle n'a pas dû effacer à Aramon le culte topique, car la continuité toponymique au Moyen Âge et à l'époque moderne (Germer-Durand 1868,9) indique sans aucun doute que sa vitalité fut préservée. Mais l'acte de Licinia Acceptilla, qu'elle soit originaire de la zone de Collias ou de celle d'Aramon, montrerait peut-être le rayonnement 
et l'attractivité acquis par le sanctuaire de l'Ermitage, ainsi que sa capacité à faire venir dans son panthéon les divinités du pays environnant et à leur assurer bon accueil. C'est une question, posée depuis longtemps, sur laquelle il conviendra de revenir plus bas.

Le texte précise que c'est à la suite de son vœu que Licinia Acceptilla a offert de ses propres deniers le portique dont les raisons de l'érection étaient signalées dans l'inscription. Ce type de construction, emprunté à l'architecture gréco-romaine, était régulièrement présent dans les sanctuaires gallo-romains, dans lesquels il offrait aux visiteurs un abri contre la pluie et le soleil ${ }^{14}$, mais des portiques et édicules présumés à vocation cultuelle se rencontrent dès l'âge du Fer, particulièrement en Basse Provence et dans le Gard (Arcelin, Gruat 2003, 201-202, fig. 110). À noter en particulier, la présence d'un portique tardo-hellénistique du début du $\mathrm{I}^{\mathrm{er}} \mathrm{s}$. av. J.-C. aux abords de la Fontaine de Nîmes (Guillet et al. 1992; Sauvage 1992) et le portique dorique situé à l'entrée du sanctuaire de Glanum, qui remplace, dans la seconde moitié du $\mathrm{I}^{\mathrm{er}} \mathrm{s}$. av. J.-C., l'édifice hypostyle destiné à l'accueil des pèlerins (Roth 1992, 52-53).

Très vraisemblablement à l'Ermitage, ce portique n'était qu'adventice; il est difficile d'admettre qu'il aurait été édifié sans qu'ait existé déjà, pour les divinités présentes, quelque(s) construction(s) en vue de les abriter ${ }^{15}$. Même s'il est difficile d'en retrouver la traduction matérielle par des traces archéologiques, on doit conclure à l'existence d'un lieu de culte monumentalisé et, du point de vue architectural, à une disposition évolutive, selon des modèles déjà répertoriés: l'exécution d'un vœu ostentatoire comme l'était celui de Licinia Acceptilla accentuait cet aspect des choses, mais il était du point de vue topographique plutôt périphérique. De plus, si le dieu Aramo pénétrait, ou avait pénétré dans le lieu sacré, tout en venant de l'extérieur, d'autres divinités devaient avoir une antériorité certaine. Même considérée isolément, cette inscription conduit aussi à réfléchir à l'aire du rayonnement du sanctuaire et aux modalités de la constitution du panthéon qui s'y trouve attesté. Nous reviendrons sur le sujet plus loin.

\section{L'ABONDANCE DOCUMENTAIRE DE L'ÉPOQUE IMPÉRIALE}

\subsection{Un autel dédié à Jupiter}

Le mieux conservé des monuments votifs est un autel connu depuis longtemps (fig. 14): il est mentionné par G. Guiran, puis, également, dans la correspondance de J.-Fr. Séguier qu'avait mise en évidence et utilisée Eugène Germer-Durand: CIL, XII, 2972 et add.; HGL, $\mathrm{XV}, 452 ;$ IAN, 214. Il fut remis en honneur par ce dernier ainsi que par A. Allmer. Il était alors engagé dans le bâti, puisque A. Allmer peut écrire, à propos de son emplacement: «dans le mur à l'angle de l'abside de la chapelle, à l'extérieur» (Allmer 1880, 132-133, n 157). Le document se trouve à présent au Musée archéologique de Nîmes, mais est également entré dans ses collections après la réalisation du Corpus, en sorte que O. Hirschfeld n'a pas pu vérifier lui-même le texte qui était gravé.

La base et le couronnement de cet autel sont assez bien préservés. Ils lui donnent une belle majesté (154 x 53 x $51 \mathrm{~cm}$ ), relevée par la sculpture d'une roue à huit rayons ${ }^{16}$ sur le dé, qui constitue le champ épigraphique $(102 \times 47 \mathrm{~cm})$. L'inscription a été gravée assez maladroitement, car on a tenté d'en insérer tout le texte entre le sommet de la roue et la moulure qui fait la transition entre le dé et le couronnement. Les lettres ont de 3,5 à 3,7 cm de hauteur (sauf le T de la ligne $3: 4,2 \mathrm{~cm}$ ).

La lecture de la ligne 2 a été la plus problématique. Dans les plus anciennes copies on laissait dans l'incertitude la partie centrale:CORI....EDENS chez G. Guiran et J.-Fr. Séguier. Puis il est apparu que l'on pouvait proposer des lectures ou des restitutions plus complètes. C'est ainsi qu'Eugène Germer-Durand, sans avoir vu l'inscription, proposait, à partir de la copie qui avait été transmise à J.-Fr. Séguier, de lire CORI[OL]EDENSES ${ }^{17}$. Puis, grâce à l'examen direct du texte, on proposa une lecture plus complète du nom de ce peuple. Auguste Allmer, informé par G. Charvet, reproduisait la lecture CORIOSSEDENSES, non sans hésiter toutefois, puisqu'il précisait: «les lettres oss, au milieu de la seconde ligne, frustes et peu certaines, surtout la seconde s,

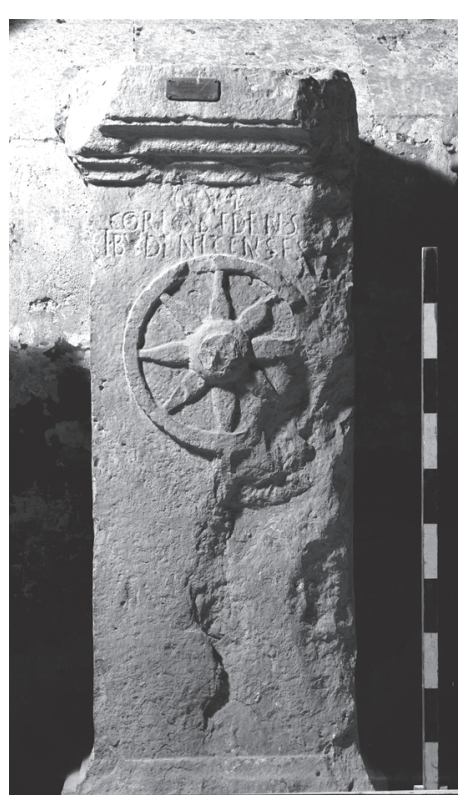

Fig. 14:Autel dédié à Jupiter par deux communautés, les Coriobedenses et les Budenicenses (cliché Foliot-Réveillac, Centre Camille-Jullian). 
qui est peut-être un $\left.\mathrm{B}^{18}{ }\right\rangle$. Mais ce fut la lecture que reproduisirent les recueils d'inscriptions: O. Hirschfeld d'abord, dans le CIL, XII, 2972 et add., lecture confirmée dans l'index des agglomérations et des peuples (p.933, même si une astérisque vient signaler la difficulté de lecture) et dans la présentation du pays entre Uzès et Nîmes (p. 372); puis les auteurs de l'Histoire générale de Languedoc $(H G L), \mathrm{XV}, 452$, enfin ceux du recueil intitulé Inscriptions antiques de Nimes (IAN), 214. Dans ces deux derniers ouvrages on ne dissimulait pas les doutes ou les hésitations: «lecture de la seconde ligne peu certaine: CORIOSSEDENS, CORIOSBEDSENS, CORIOBEDENS ». La lecture qui semble la meilleure, après révision de l'inscription est CORIOBEDENS[ES].

En principe, la lecture de la ligne 3 est moins problématique. Au début, comme on s'y attendait, on peut lire la conjonction de coordination ET. Cette ligne, légèrement décalée à droite par rapport à la précédente, pouvait courir de bout en bout du champ épigraphique: il est difficile d'envisager que l'on ait voulu graver le premier mot dès le bord du champ épigraphique à droite, si la ligne ne devait pas aller jusqu'à l'autre bord. Aussi, après les lettres formant le mot bvdenicenses, on estimera qu'une ou deux lettres pouvaient manquer: s'agirait-il de celles qui sont caractéristiques d'un vœu $(\mathrm{v}, \mathrm{s})$ ?

On lira donc:

Iovi

Coriobedens[es]

et Budenicenses [--]

«À Jupiter, les communautés des Coriobedenses et des Budenicenses».

Deux petites communautés locales se sont ainsi associées pour honorer Jupiter. On rapprochera les Budenicenses, de Mars Budenicus, dont l'existence a été révélée par une inscription examinée ci-dessous. Jupiter apparaît comme un dieu majeur du lieu, et c'est à lui que l'on donnera sans aucun doute la prééminence, en le considérant comme dieu topique. Sans aucun doute, sous son nom se cache une divinité indigène dont l'identité nous échappe.

\subsection{Un pilier : autel ou support d'offrande?}

Cet autre document, longtemps vu à l'Ermitage de Collias, a été transporté, comme la plaque relative au portique de Licinia Acceptilla, au Musée archéologique de Nîmes, mais il y est parvenu également après la rédaction du Corpus, car O. Hirschfeld ne l'a pas vu de lui-même $(C I L, \mathrm{XII}, 2973$ et add. =ILS, 4549; HGL, XV, 1511; IAN, 549; voir $C A G, 30-2,331-332$, notice $\left.085, \mathrm{n}^{\circ} 9^{*}\right)$. Il s'agit, dans l'état actuel, d'un bloc de forme très élancée (h. 190, 1.38, ép. $36 \mathrm{~cm}$ ). On le considère habituellement comme un autel, mais il peut s'agir, comme dans le cas précédent, du support d'une offrande, en forme de pilier (fig. 15). Le couronnement, haut de $18 \mathrm{~cm}$, a été abattu sur la face principale, à droite et à l'arrière, mais on distingue, à gauche, la liaison entre celui-ci et le dé sur lequel se trouvait le champ épigraphique, qui s'effectue par une élégante moulure. Il en va de même pour la transition entre la base, haute de $27,5 \mathrm{~cm}$ et le dé.

Le texte est connu depuis longtemps. Jean-François Séguier, lors de son grand voyage européen en compagnie de Scipione Maffei, en avait reçu connaissance par une lettre d'un de ses frères, le chanoine de Saint-Gilles, que E. Germer-Durand a retrouvée dans ses papiers. Ce dernier a même estampé l'inscription, précisant alors qu'en son temps le bloc «formait la seconde marche de l'autel de la chapelle de l'ermitage» (Germer-Durand 1875, 264, 267, 268). La révision du texte permet de vérifier que les mutilations que l'on constate actuellement existaient dès le XVIII ${ }^{\mathrm{s}} \mathrm{s}$ : elles avaient été provoquées par la réutilisation du bloc.

À la ligne 2, on a d'abord envisagé de lire le mot BVDENIO. Ce point de vue, adopté initialement par Eugène Germer-Durand, s'est avéré fragile et a bien vite été abandonné. On a préféré lire BVDENIC[O], car la dernière lettre conservée est bien C. Puis il fallait insérer, à la fin de la ligne, la lettre o: mais peut-être s'inscrivait-elle dans le C, ou bien avait-elle des dimensions réduites. Il est inutile de vouloir restituer le qualificatif du dieu sous la forme Budenicensis, comme l'aurait souhaité A. Allmer (1880, 133-134, n 158). À la ligne 4, il n'apparaît pas possible de lire la moindre trace du I final du mot Severi. Les lettres sont progressivement décroissantes: $1.1: 7 \mathrm{~cm}$ $(\mathrm{I}: 8 \mathrm{~cm}) ; 1.2: 5,2 \mathrm{~cm} ; 1.3: 5 \mathrm{~cm} ; 1.4: 4,2 \mathrm{~cm} ; 1.5: 4$, mais 4,5 pour le premier $\mathrm{I}$.

On lira donc:

Marti

Budenic[o]

Gratus

Sever[i]

filius

«À Mars Budenicus, Gratus, fils de Severus »

On reviendra plus loin sur l'identité de la divinité. Mais le personnage mentionné, qui offre l'autel ou bien le pilier surmonté d'une offrande destinée à ce dieu Mars Budenicus, porte une dénomination pérégrine, qui pourrait indiquer qu'il appartient au fond indigène de la population. Néanmoins, tous les éléments de sa dénomination, l'idionyme comme le patronyme, sont parfaitement latinisés (Christol, Deneux 2001), en sorte que celle-ci a perdu toute trace de réminiscence locale, ce qui n'était pas tout à fait le cas de Licinia Acceptilla. Tout au plus, comme pour cette dernière, relèvera-t-on que les noms qui apparaissent, celui du fils (Gratus) autant que celui du père (Severus), sont bien attestés dans l'anthroponymie de la colonie de Nîmes, et donc bien acclimatés dans la population 
locale, sans que l'on puisse envisager qu'ils traduiraient un nom plus proprement celtique ${ }^{19}$. Quant à la date du texte, par comparaison avec la gravure de l'inscription de Licinia Acceptilla, on peut proposer une date à peu près identique, soit la seconde moitié du I ${ }^{\text {er }}$ siècle ap. J.-C. Le monument est de belle facture, il peut indiquer une certaine aisance, sans pour autant orienter vers la partie de la société la plus relevée.

\subsection{Un monument votif :}

\section{autel ou support d'une offrande?}

Un autre document doit être considéré (fig. 16). Il est actuellement conservé à la mairie de Collias $(C I L$, XII, 2974 = ILS, 4662; HGL, XV, 1512). Il s'agit, dans l'état actuel, d'un bloc de forme élancée (h. 123, 1.27 , ép. $26 \mathrm{~cm}$ ), qui peut correspondre à un autel largement retaillé. Il peut s'agir aussi du support d'une offrande. Le couronnement a été abattu, mais on distingue, à droite, la liaison entre celuici et le dé sur lequel se trouvait le champ épigraphique; sur le côté gauche aussi, une retaille a affecté verticalement l'ensemble du couronnement et du dé, avec de larges éclats.

Le texte est connu depuis longtemps. L'autel demeurait inaccessible. On se fondait sur les indications de l'érudit nîmois G. Guiran. Il indiquait la provenance: in aedicula divae Vallensis seu de Valle, prope Coliacum, in arula oblonga (Germer-Durand 1875, 262; Hirschfeld, ad CIL, XII, 2974). Et c'était sa lecture, légèrement inexacte, qui était considérée cependant comme la meilleure, reléguant dans l'apparat critique les autres témoignages, d'où $C I L$, XII, 2974 et $H G L, X V, 1512$. Elle avait conduit les auteurs de $H G L, \mathrm{XV}, 1512$, à considérer qu'il manquait peut-être une ligne au début du texte, concernant le nom d'un éventuel dédicant, alors que $\mathrm{O}$. Hirschfeld estimait que la copie de G. Guiran fournissait l'intégralité du texte. Toutefois, ce dernier n'hésitait pas à la corriger, à propos du premier nom de la divinité. Il écrivait SVL[e]viAE, appelant donc la divinité Sulevia Idennica Minerva, comme l'indique son index ${ }^{20}$, puis comme l'indique H. Dessau qui à son tour a reproduit l'inscription (ILS, 4462, et index, vol. III, 1, p. 545 et 552). La redécouverte de celle-ci, avant même son transport à la mairie de Collias, a permis de reprendre l'édition du texte.

Il ne peut plus y avoir d'hésitation sur la première ligne. La lecture de G. Guiran donne un texte complet, puisque l'on peut voir la trace du contact entre dé et couronnement, à quelques centimètres au dessus de la première ligne. De plus, la correction par O. Hirschfeld de la lecture SVLIVIAE en Suleviae est pleinement justifiée. La légère erreur de lecture de G. Guiran ou de son correspondant se comprend parfaitement, car les barres horizontales des lettres sont très finement gravées $(\mathrm{E}, \mathrm{T})$. En revanche, à la ligne 2, il faut renoncer à la lecture Idennica, admise

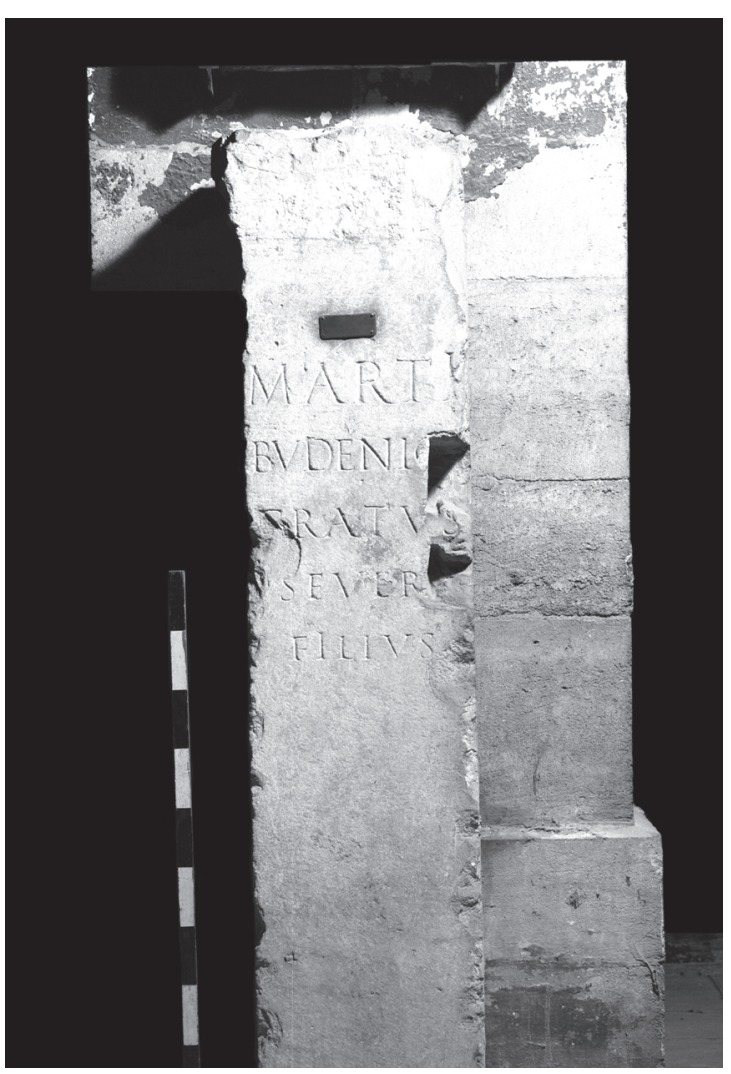

Fig. 15:Autel ou support d'offrande dédié à Mars Budenicus par Gratus, fils de Severus

(cliché Foliot-Réveillac, Centre Camille-Jullian).

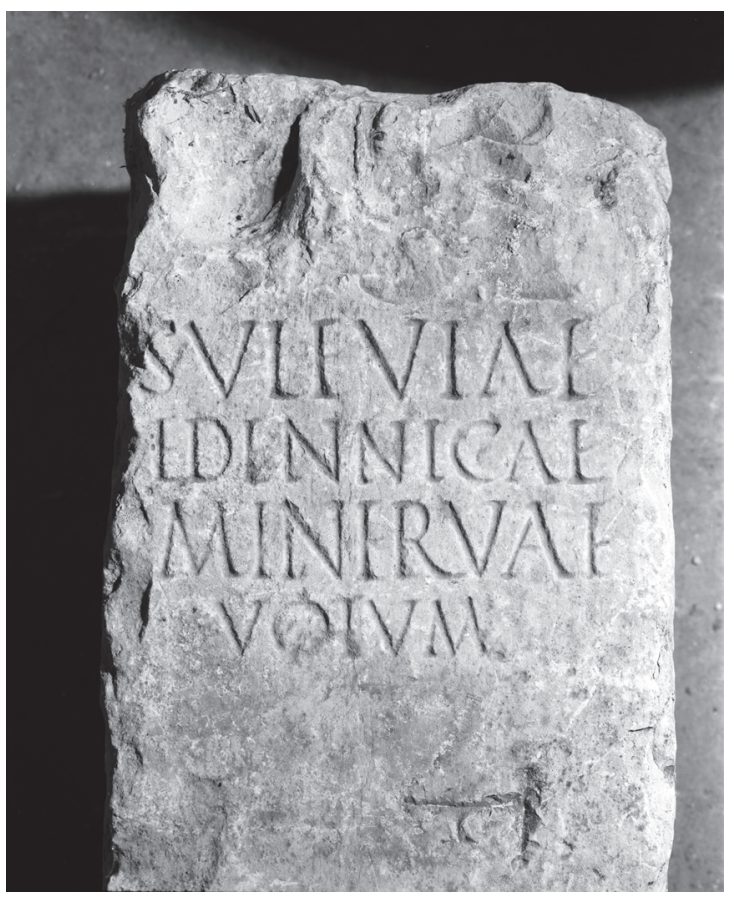

Fig. 16:Autel ou support d'offrande dédié à Sulevia [L]edennica, Minerve (cliché Foliot-Réveillac, Centre Camille-Jullian). 
par tous les auteurs. Comme nous l'avons indiqué lors de la préparation de la $C A G$ (d'où $C A G, 30-2,332$, notice $085, n^{\circ} 9^{*}$, fig. 342), on peut hésiter entre deux lectures: Edennicae et [L]edennicae. La première lecture est celle qui transcrit le texte visible. Mais il convient de s'interroger quelque peu sur la réalisation de la mise en page et sur le travail du graveur. On relève que les lignes 1,2 et 3 , les plus longues, ne sont pas, du côté droit, parfaitement à l'aplomb, et que le graveur, s'il avait été suffisamment habile, aurait pu parfaitement commencer et terminer à l'aplomb les trois premières lignes. On relève aussi que la ligne 2 est en plus petits caractères que celles qui l'encadrent (1. 1 : entre 3,8 et $4 \mathrm{~cm} ; 1.2$ : entre 3 et 3,$1 ; 1.3$ : entre 3,6 et 3,$8 ; 1.4$ : entre 2,4 et $2,7 \mathrm{~cm}$ ). C'est aussi celle qui comporte le plus grand nombre de lettres. On peut donc estimer que le graveur a réduit la taille des caractères pour faire entrer un mot plus long que les autres. Dans ce cas, de la même manière que le $\mathrm{E}$ final déborde un peu à droite, ne faut-il pas envisager aussi que la lettre initiale devait déborder légèrement à gauche? Il nous semble donc possible d'envisager l'existence d'une première lettre, assez étroite, venant précéder celles qui sont conservées au début de la deuxième ligne. Nous proposerons de lire [L]EDENNICAE.

En somme, le texte se présenterait de la façon suivante:

Suleviae

[L]edennicae

Minervae

votum

«À Sulevia Ledennica, Minerve. Autel installé selon un vœu»

Ce monument votif fait connaître une divinité du sanctuaire. Elle a été identifiée à Minerve. Le mot Ledennica se termine par le suffixe d'appartenance -icus/ica. Lorsque l'on dénommait cette divinité Idennica, on la rattachait au village de Seynes (anciennement Eysennes) et au petit ruisseau qui y prend sa naissance ${ }^{21}$. La lecture qui vient d'être proposée rapprocherait plutôt de Letinno, divinité qui est connue par une inscription de Lédenon (CIL, XII, $2990=I L S, 4679) ; H G L, X V, 1479 ; I A N, 48)$, et qui, pour cette raison, a aisément été rapprochée de ce toponyme ${ }^{22}$. Or, à vol d'oiseau, Lédenon se trouve à moins de $4 \mathrm{~km}$ de l'Ermitage de Collias. Si cette interprétation s'impose, on retrouverait ici, également, le déplacement d'une divinité vers ce sanctuaire et son installation, sans que vraisemblablement le lieu de culte originel ait eu à subir une transformation. Le terme [L]edennica indiquerait ainsi l'origine de cette divinité en un lieu différent de son sanctuaire principal.

La seule incertitude concerne la destination du bloc retaillé. Les remaniements ont fortement altéré le couronnement et ont fait disparaître d'éventuels éléments significatifs. Aussi ne peut-on pas exclure totalement qu'il se serait agi d'un socle supportant une offrande, elle aussi promise par vœu. Mais ceci ne change rien à la façon d'envisager la présence de cette divinité assimilée à Minerve. Les caractéristiques de la gravure des lettres, qui est en général bien plus souple que dans l'inscription précédente, conduiraient à placer cette inscription au $\mathrm{II}^{\mathrm{e}} \mathrm{s}$. ap. J.-C.

\subsection{D'autres nouveaux documents}

L'abbé Bayol, qui avait organisé un musée lapidaire sur le site, signalait la présence de colonnes de divers modules dont deux en marbre gris, et d'un torse en grès couvert d'une dalmatique (Bayol 1980, 14, 16); il notait aussi le remploi d'un monolithe en pierre de Barutel dans l'ermitage (Bayol 1980, 15), mais ces documents, aujourd'hui disparus, n'étaient pas forcément d'origine antique. D'autres, qui n'avaient jamais été signalés, illustrent cependant que le nombre des remplois était important. Ainsi, la partie inférieure d'un autel orné d'une roue (fig. 17) a été vue lors des visites de 2004 et de 2005 au milieu du transept ${ }^{23}$. C'est un nouveau témoignage du culte de Jupiter. L'examen attentif des murs de l'église a également permis de repérer un bloc de la porte intérieure que ses dimensions rattachent aux documents de la série étudiée: il a conservé une hauteur de $60 \mathrm{~cm}$, une base aux moulures rabattues de 41 sur $51 \mathrm{~cm}$ et un dé large de $31 \mathrm{~cm}$ (fig. 18). On peut également penser que les deux impostes moulurées qui supportent, au sud, les arcs du transept, sont d'origine antique (fig. 19): G. Durand avait observé que les impostes étaient chanfreinées au nord et moulurées au sud, et que ces dernières semblaient inadaptées à leur support; elle pensait à des remplois de l'église du $\mathrm{XI}^{\mathrm{e}} \mathrm{s}$. (Durand 1992, 139 et n. 17), mais leurs moulures s'apparentent bien à celles de cippes antiques.

\subsection{Des monuments votifs dans la tradition régionale}

Les monuments en pierre de taille les mieux conservés se caractérisent par leur hauteur qui les distingue du modèle classique de l'autel sacrificiel. On notera, en particulier, que la dédicace à Minerve est inscrite sur un bloc dont les dimensions sont pratiquement les mêmes que celles du pilier des Mères; elle ne s'en distingue que par un couronnement mouluré. Du même type est la dédicace à Mars Budenicus, sur un pilier encore plus grand. Et si l'autel à Jupiter a une forme moins élancée et s'il comporte une base et un couronnement moulurés, il peut également s'inscrire dans la tradition des piliers votifs, particulièrement fréquents en Languedoc oriental et en basse Provence, et qui, dès les $\mathrm{VI}^{\mathrm{e}}-\mathrm{V}^{\mathrm{e}} \mathrm{s}$., se distinguent, parmi les cippes ou bétyles, par leur hauteur, généralement comprise entre 1,5 et 2,25 m (Arcelin et al. 1992, 188, fig. 4 et 195). À partir du III ${ }^{\mathrm{e}}$ s. av. J.-C. au moins, ils sont souvent moulurés, et, 


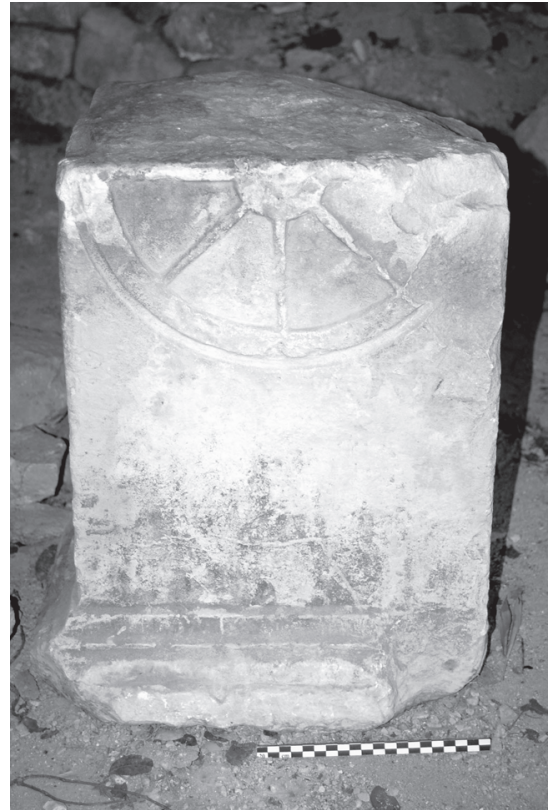

Fig. 17: Autel orné de la roue de Jupiter (cliché J.-L. Fiches).

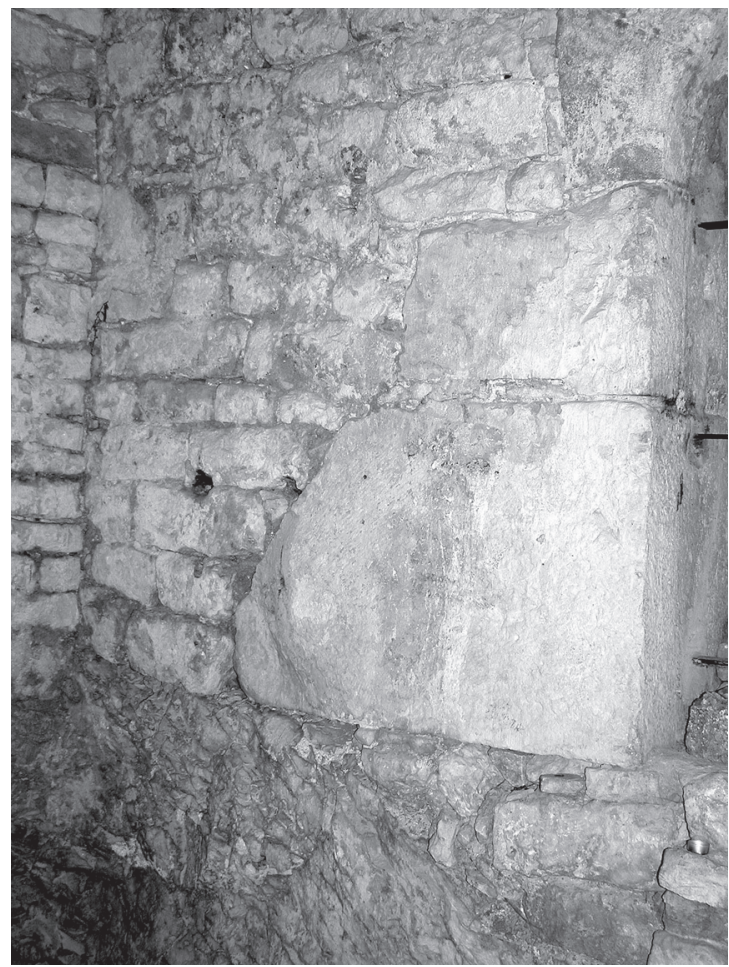

Fig. 18: Deux blocs de la porte intérieure entre la nef et le transept sont des remplois antiques; par leurs dimensions il

s'agit d'un autel funéraire (en bas) et d'un autel votif ou support d'offrande (en haut) (cliché J.-L. Fiches). dans cette série, on retiendra en particulier les quatre cippes à base et couronnement débordants qui ont été trouvés anciennement près de la source de l'Arcoule à Paradou (B.-du-Rh.); la présence d'une mortaise dans la partie sommitale des trois qui ont été entièrement conservés (h. 0,97 à $1,28 \mathrm{~m}$ ) montre qu'il s'agissait de socles supportant un objet; ils ont été interprétés comme des monuments funéraires en raison de la découverte, sur le site, d'une nécropole du $\mathrm{I}^{\mathrm{er}} \mathrm{S}$. av. J.-C. (Arcelin 1979) mais on ne peut exclure qu'ils aient fait partie d'un ensemble cultuel lié à la source.

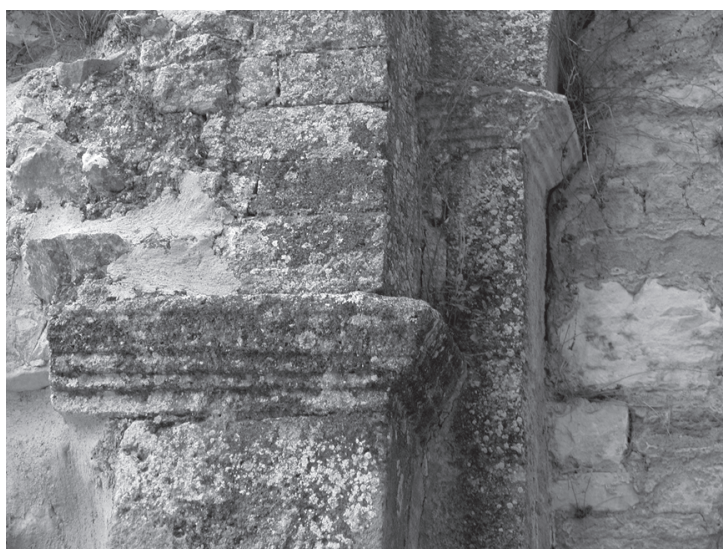

Fig. 19: Les deux impostes moulurées, inadaptées à leur support, sur lesquelles retombent, au sud, les arcs du transept, sont vraisemblablement des remplois de cippes antiques (cliché J.-L. Fiches).

\section{LE PaNTHÉON dU SANCTUAIRE de Collias}

\subsection{L'identification d'un sanctuaire}

L'ample groupement documentaire est apparu comme un élément significatif, propre à susciter une explication de la nature du site. Eugène Germer-Durand, sur la foi de l'inventaire des inscriptions, qu'il établissait pour la première fois, estimait qu'il constituait un témoignage sur la résistance du paganisme, «idolâtrie rustique» qu'aurait vaincue l'ermite Vérédème: "Comment expliquer la réunion, sur un même point, dans le fond de cette combe étroite et déserte, encore aujourd'hui presque inaccessible, de tant d'autels votifs en l'honneur de divinités païennes, Jupiter, Minerve, Mars Budenius, Sulivia Idennica, Aramon, sans compter ceux qui ont été détruits et ceux qui pourront se révéler un jour? Bien que l'aspect des lieux semble se prêter difficilement à cette hypothèse, il faut nécessairement admettre qu'il y avait là un centre religieux pour les peuplades arécomiques, et que les pagani des environs y continuaient leur culte superstitieux, alors même que le christianisme était définitivement établi dans 
les villes où siégeaient des évêques, et commençait à pénétrer dans les centres de population importants ou situés sur le parcours des voies romaines. La combe inabordable de Laval était alors devenue le refuge des partisans attardés des pratiques idolâtriques; et lorsque, dans la première moitié du VII ${ }^{\mathrm{e}} \mathrm{s}$., S. Vérédème choisit, pour y fixer sa demeure «et y mener la vie angélique», la grotte qui est devenue la chapelle Notre-Dame, il ne venait pas seulement chercher en ce lieu la solitude; il venait surtout renverser ce qui restait encore de l'idolâtrie rustique; et c'est en signe de cette victoire que lui, ou les anachorètes ses successeurs, jetèrent, dans les fondements de la modeste chapelle qu'ils élevèrent au vrai Dieu, les autels païens dont quelques-uns ont été recueillis» (Germer-Durand 1875, 268-269). Ce point de vue met en évidence les caractéristiques topographiques du site, la diversité des témoignages épigraphiques, la juxtaposition de pratiques cultuelles antiques et d'un lieu de culte chrétien.

La diversité des témoignages épigraphiques est analysée selon deux orientations. L'une, qui n'est peut-être pas suffisamment argumentée du point de vue chronologique, parvient à dégager du regroupement des documents le constat qu'existait «un centre religieux». Son rôle se serait peut-être renforcé avec le temps, si même cette fonction de «centre»n'avait pas été suscitée, exploitée et étendue à l'occasion, dans un contexte de résistance religieuse durant l'Antiquité tardive. L'autre orientation, qui apparaît plus nettement dans le commentaire, s'appuie sur la superposition des témoignages cultuels et sur l'importance symbolique que revêtent les remaniements et les réutilisations: perte de sens pour les documents anciens, qui sont remployés, imposition d'une nouvelle signification par la construction d'un édifice qui marque l'hégémonie de la nouvelle religion.

Auguste Allmer, pour sa part, ne reprit que l'essentiel de cette analyse, et sous une forme plus froide et distanciée: «Là sont groupées cinq inscriptions romaines [il s'agit aussi d'inscriptions funéraires], dont la présence dans ce désert autorise à supposer la préexistence d'un sanctuaire païen à la chapelle chrétienne» (Allmer 1880, 132). Il approfondissait ensuite ses observations, lors de la publication de l'inscription de Licinia Acceptilla: «À cet Ermitage se trouvent déjà réunis, on se le rappelle, plusieurs autres autels à des dieux que leurs noms identifient avec des localités assez éloignées, et il est cependant peu concevable que ces pierres aient été apportées de loin en un lieu si désert et si peu accessible» (Allmer 1881, 189).

C'était inviter à dégager tout le sens de ce rassemblement d'inscriptions, et, pour comprendre l'organisation de ce panthéon, ne pas omettre la dimension géographique qui apparaît de façon remarquable.

\subsection{L'organisation du monde divin}

Le dieu appelé Jupiter doit être considéré comme le dieu principal, une des divinités d'origine de ce sanctuaire. Ce n'est peut-être pas seulement parce qu'il faut lui attribuer le plus grand nombre de témoignages explicites, par le texte et par l'image. Deux, au total, sont sûrs: un autel à la roue anépigraphe, et l'autel à la roue dédié par les Coriobedenses et les Budenicenses.

On pourrait même être tenté de rattacher à ces deux témoignages, bien localisés à l'Ermitage de Collias, un autre document, actuellement conservé au château de Saint-Privat, sur la commune de Vers-Pont-du-Gard: $C I L$, XII, 2981 et add. $=H G L, X V, 1504^{24}$. Le lieu de conservation se trouve sur la rive droite du Gardon, à quelques kilomètres en aval de Collias. L'autel (fig. 20) avait été remployé dans la construction d'une petite chapelle romane qui existait encore en 1554. Il est remarquable par ses dimensions (h. $91 \mathrm{~cm}, 1.36 \mathrm{~cm}$, ép. $29 \mathrm{~cm}$ ), ce qui le rapproche des autres autels ou monuments étudiés ci-dessus, bien différents des petits autels votifs, parfois minuscules, épigraphes ou anépigraphes, que l'on trouve couramment dans les sanctuaires. Il constitue un témoignage original sur le culte impérial: au lieu d'associer simplement le dieu Jupiter et l'empereur, désigné d'une façon générique, il met en relief une caractéristique religieuse du divin, la sanctitas, en utilisant un substantif qui mérite d'attirer l'attention ${ }^{25}$. Le texte se lit ainsi ${ }^{26}$ :

Sancti-
tati
Iovis et
Augusti
sacrum
[-] Lucili[us]
Cestio

« À la sainteté de Jupiter et d'Auguste; (Lucius) Lucilius Cestio a consacré (cet autel)»

En associant Jupiter et le prince, sous ce vocable, c'est le second nommé, le prince du moment, qui voit sans aucun doute sa nature rehaussée et exaltée. En effet, évoquer la sanctitas d'une personne, ou lui attribuer l'épithète sanctus/sancta, c'est mettre l'accent sur le caractère exceptionnel de ses vertus, et lui attribuer une nature hors du commun (Fugier 1963, 278-283). Ajouter au nom d'un dieu ou d'une déesse une telle épithète, c'était, en énonçant le nom, confirmer sa nature divine (Fugier 1963, 270-276). Ainsi, associer à la sanctitas de Jupiter celle du prince c'était attribuer au prince toute la force qu'en d'autres circonstances les dieux seuls pouvaient manifester, c'était lui reconnaître une énergie que seule pouvait éclairer la comparaison avec celle des dieux. On n'éloignera donc pas du dossier ce témoignage remarquable sur les formes que prit le culte impérial dans les 

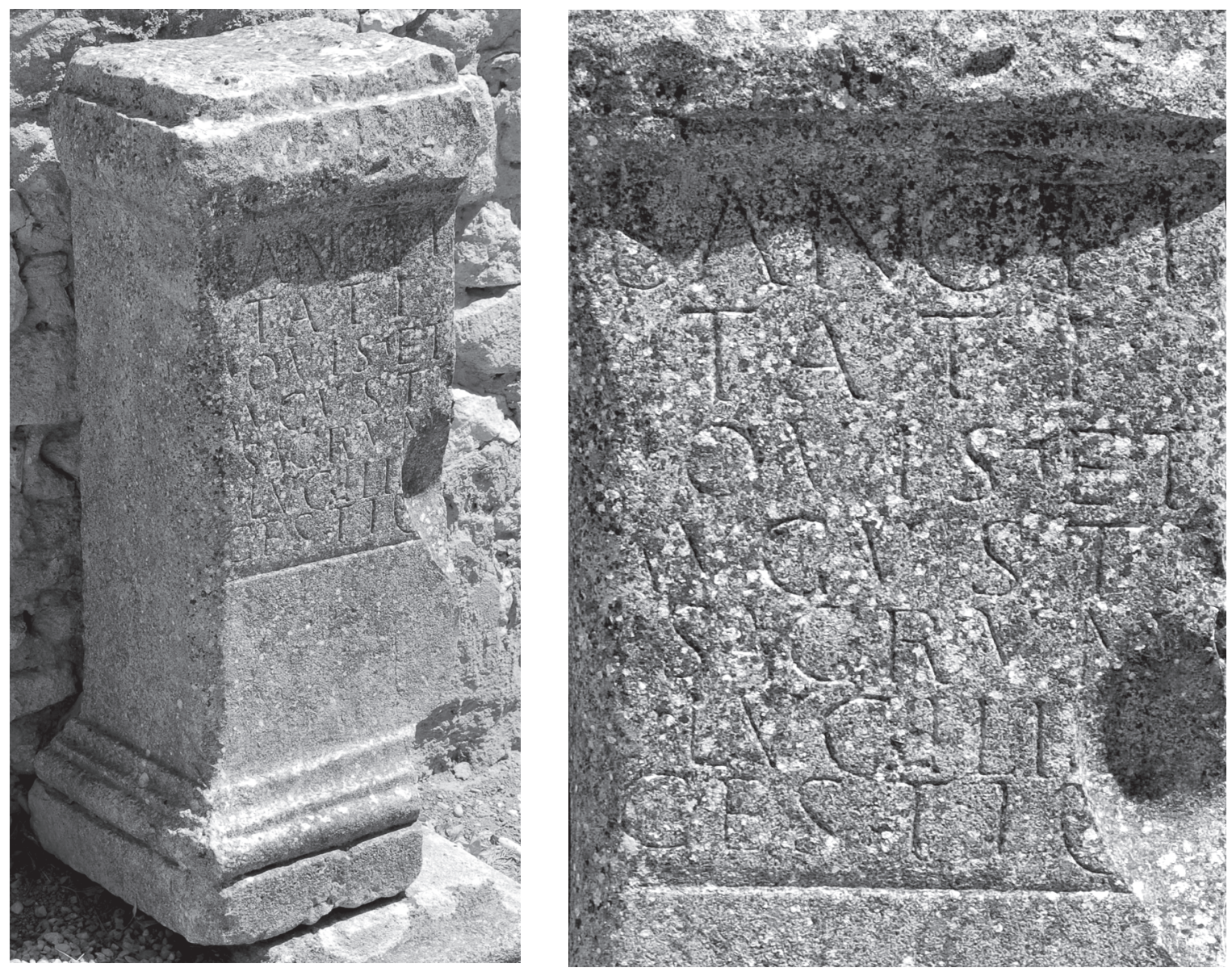

Fig. 20: Conservé au château de Saint-Privat (Vers-Pont-du-Gard), autel consacré par L. Lucilius Cestio à la sainteté de Jupiter et de l'empereur (cliché J.-L. Fiches).

cités de Gaule méridionale, à partir du premier quart du II $\mathrm{s}$. ap. J.-C. (Chastagnol 1999).

Le culte de Jupiter à la roue est attesté à plusieurs reprises dans le territoire de la cité de Nîmes: à Lansargues (CIL, XII, 4179 et add.; HGL, XV, 1855; $C A G, 34-3,194$, notice $\left.127, \mathrm{n}^{\circ} 25\right)^{27}$, à Marsillargues (CIL, XII, 4172; HGL, XV, 1842; CAG, 34-3, 249-250, notice $\left.151, \mathrm{n}^{\circ} 3\right)^{28}$, deux fois à Tresques, d'abord sur un autel à la roue (CIL, XII, 2752; HGL, XV, 1548;CAG, $30-3,703-704, n^{\circ} 331$, notice 10 , fig. 871 ) puis sur un pilier (CIL, XII, 2753; HGL, XV, 1547; CAG, 30-3, 711, notice $\left.331, \mathrm{n}^{\circ} 25\right)^{29}$, ainsi qu'à Clarensac où le dieu est associé à la Terre Mère (CIL, XII, 4140 et add. ; HGL, XV, $4=1781 ; C A G, 30-2,324$, notice $\left.082, \mathrm{n}^{\circ} 2\right)^{30}$ et à Psalmody (associé à Silvain; $A E, 1919,84$; ILGN, 516; $C A G, 30-3,635$, notice $\left.276, \mathrm{n}^{\circ} 2\right)^{31}$. Ce n'est peut-être pas partout le même dieu, mais dans cet inventaire des témoignages, on relève incontestablement des divinités qui pouvaient aisément être identifiées à la même divinité romaine, le nom de Jupiter effaçant quelque peu leur particularité, qu'elle soit fonctionnelle ou topique, comme le faisaient aussi, dans d'autres cas, Mars ou quelque autre divinité du panthéon romain.

$\mathrm{Si}$, grâce aux documents, nous avons suffisamment de renseignements sur le panthéon local, c'est à ce dieu que l'on accordera la primauté sur le lieu de culte, à tout le moins l'antériorité d'installation. En effet, si les Budenicenses l'honorent collectivement, il faut remarquer qu'ils ont pour eux-mêmes une divinité, dont l'origine a été précisément signalée dans le texte qui a été gravé: Mars Budenicus. Le qualificatif Budenicus marque précisément l'appartenance ou bien la relation avec le petit groupement humain qui est protégé. C'est pourquoi l'absence de cette indication de rattachement peut conférer à ce Jupiter la primauté. C'est lui qui accueille les divinités venues d'ailleurs et qui les admet dans son entourage.

On peut estimer qu'il avait à ses côtés un groupe de divinités féminines, «les divinités de la source d'en bas». Elles seraient à rapprocher des Mères gauloises, bien attestées par ailleurs. On rappellera que dans le sanctuaire 
du Plateau des Poètes, à Béziers, à côté de Mars et de Ricoria, qui paraissent dominer l'activité religieuse du site, se trouvent des divinités attestées au pluriel, les Menmanduti et les Digenes (CIL, XII, 4223 et 4216; Christol 2003a, 412-413, 415-417), tout comme à Cadenet, dans la cité d'Aix-en-Provence, les Caudellenses accompagnent Dexsiva (CIL, XII, $1064=I L N$, Aix-enProvence, 222).

La dénomination des autres divinités montre leur entrée dans le lieu de culte. Nous l'avons déjà précisé en ce qui concerne Aramo. Si l'on doit rattacher Mars Budenicus aux Budenicenses, et faire de ces derniers les membres d'une communauté voisine de Collias, à l'instar des Coriobedenses, qui devraient en être également très proches par leur localisation, comme invite à l'envisager leur association établie dans l'inscription, il en va de même de Sulevia [L]edennica Minerva. On a vu plus haut les raisons de tenter de la rattacher au dieu Letinno et au toponyme Lédenon. Ces deux divinités n'ont pas subi de transfert de culte, mais, par la volonté des communautés auxquelles elles étaient liées, elles ont vu leur culte s'établir dans un autre lieu de la cité sans vraisemblablement s'effacer dans le lieu d'origine. En quelque sorte, le sanctuaire de Collias et les divinités qui s'y trouvaient ont été accueillants aux dieux voisins, en même temps qu'ils recevaient la visite et l'hommage de leurs dévots: rayonnement du sanctuaire et attractivité sont les éléments qui se dégagent du dossier. Au XIX ${ }^{\mathrm{e}} \mathrm{s}$., on avait cherché à localiser les Budenicenses dans la région d'Uzès et dans la moyenne vallée du Gardon, mais aujourd'hui les références à Aramon et à Lédenon invitent à considérer la voie Nîmes-Avignon comme l'un des principaux vecteurs de ce rayonnement.

On remarquera aussi que se mêlent étroitement les éléments proprement romains et les éléments proprement autochtones. Les noms des divinités en témoignent. Aramo se place nettement du côté indigène, alors que Jupiter se trouve sur le pôle opposé. En revanche Mars Budenicus et Sulevia [L]edennica Minerva présentent des dénominations mixtes, dans lesquelles les noms romains n'ont pas encore effacé, sous un vocable uniformisateur, la diversité locale ${ }^{32}$.

En effet Mars Budenicus entre dans une belle série de divinités comparables ${ }^{33}$. Non loin d'Uzès, à Serviers-etLabaume est connu un Mars [---]gius (AE, 1903 , 363; ILGN, 394; CAG, 30-3, 689-690, notice 319, nº 5). À Nîmes, par l'épigraphie, apparaissent quelques autres attestations remarquables. On signalera d'abord, parce qu'elle renvoie à l'acte religieux d'une petite communauté au sein de la colonie de Nîmes, l'autel dédié à Mars Augustus Lacavus par les Adgentii (CIL, XII, 3084; HGL, $\mathrm{XV}, 13=451 ; \operatorname{IAN}, 5=213 ; C A G, 30-1,346$, notice 311 , inscr. $\mathrm{n}^{\circ} 3$ ). On ajoutera deux témoignages relatifs à Mars
Britovius (CIL, XII, 3082 et add.; HGL, XV, $11=468$; $I A N, 4$; CAG, 30-1, 429, notice 464 , inscr. ${ }^{\circ} 2$; ainsi que CIL, XII, 3083; HGL, XV, $12=469 ; C A G, 30-1,470$, notice 557, inscr. $\left.n^{\circ} 1\right)$. Enfin on signalera Mars Melovius (AE, 1955, 106; AE, 1959, 192-193; Christol 1990, 179, n. $5 ; C A G, 30-1,468$, notice 550 , inscr. $\mathrm{n}^{\circ} 71$ ).

On peut donc, sur le témoignage de Sulevia [L]edennica Minerva, se demander s'il n'en va pas de même pour les autres attestations de Minerve dans la cité de Nîmes. On la retrouve sous ce seul vocable dans un autre sanctuaire de la cité, au sud de Beaucaire (CIL, XII, 5884; HGL, XV, 1429; CAG, 30-2, 227, notice 032, $\mathrm{n}^{\circ} 73$; Christol et al. 2005-2006), ainsi qu'à Combas, à l'ouest de Nîmes, à deux reprises (ILGN, 385, 386; GAG, 30-2, 339-340, notice $\left.088, \mathrm{n}^{\circ} 12\right)$ : il s'agit dans ces deux cas d'un culte féminin. On ajoutera un témoignage à Garons, au sud de cette ville (ILGN, 512;CAG, 30-2, 375, notice 125): il s'agit aussi d'un culte féminin. Puis on mentionnera, dans la région de Montpellier (CIL, XII, 4185; HGL, XV, 1862; $C A G, 34-3,283$, notice $173, \mathrm{n}^{\circ} 36$ ), un cas de provenance imprécise: il s'agit aussi d'un culte féminin. Enfin, à Nîmes même, sa présence est attestée dans le sanctuaire de la Fontaine (CIL, XII, 3092 et add. ; HGL, XV, $24=$ 464 ; IAN, 11; CAG, 30-1, 262, notice 130, $\mathrm{n}^{\circ} 2$ ) sur un autel de très petites dimensions, et dans un autre cas elle est associée aux Lares augustes ainsi qu'à des divinités locales, Avicantus et Urnia (CIL, XII, 3077; HGL, XV, 23 = 426; CAG, 30-1, 271, notice 149). Néanmoins, l'exemple qui provient de l'Ermitage demeure isolé, car, si cette divinité s'était caractérisée à un moment de son histoire par la préservation d'une mémoire indigène grâce à des épiclèses, celles-ci ont régulièrement disparu. Aussi la Sulevia-Minerve de Collias présente-t-elle un grand intérêt. Sa dénomination révèle fortement sa caractéristique de divinité topique. On retiendra le rapprochement effectué par P. Finocchi $(1994,97)$ avec une Sulevia connue à Strasbourg (Petry 1982, 57-60) et considérée comme une Tutela, divinité protectrice du lieu. Elle est bien rattachée à une communauté de petites dimensions et au lieu de culte principal qui s'y trouvait par un adjectif en -icos, dans la tradition celtique. De plus, l'assimilation de la déesse locale à Minerve révèle une certaine cohérence, puisque la même association se retrouve en Bretagne, à Bath (Aquae Sulis) ${ }^{34}$.

On est donc en droit de considérer le site de l'Ermitage de Collias comme un sanctuaire religieux. Son histoire est ancienne et vraisemblablement ancrée dans la période protohistorique comme le suggère la présence de l'inscription gallo-grecque aux Mères dans un contexte (à proximité d'une source) qui invite à des rapprochements avec les sanctuaires urbains de Nîmes et de Glanum. Mais elle est plus connue par l'épigraphie que par l'archéologie car les remaniements et les constructions du culte chrétien 
ont tout supplanté et beaucoup effacé. Ainsi, on ne saurait rien des bâtiments du Haut-Empire si l'une des inscriptions ne mentionnait un portique dont la construction suppose l'existence préalable d'un temple.

Même si l'ampleur des regroupements n'est pas comparable à celle que l'on constate au sanctuaire de la Fontaine, elle demeure remarquable. À moins de $20 \mathrm{~km}$ du chef-lieu de la colonie latine, un panthéon indigène, honoré par les populations des environs, se maintenait, qui plus est en s'accroissant d'apports plus ou moins voisins, puisqu'Aramo venait lui-même d'une vingtaine de kilomètres plus à l'est encore. À la différence des autres témoignages religieux liés à une source, qui sont fréquents mais beaucoup plus modestes dans cité de Nîmes en dehors des agglomérations (Marrou 1932; Blétry-Sébé 1986), la Combe de l'Ermitage apparaît donc comme un lieu de rassemblement rural au rayonnement large - en particulier vers l'est et le sud (où se situait sans doute l'accès principal) alors que les érudits du XIX ${ }^{\mathrm{e}} \mathrm{s}$. orientaient leur quête vers le nord/nord-ouest, en direction d'Uzès et d'Alès. Ce n'était pas sans doute le seul de ces sanctuaires dont la réputation dépassait le cadre local; sur le territoire des Arécomiques et dès le $\mathrm{I}^{\mathrm{er}} \mathrm{s}$. av. J.-C., il en est un qu'on situe en bordure du Rhône au sud de Beaucaire (fig. 3), grâce à la dédicace du préteur des Volques T. Carisius, déjà connu dans un autre sanctuaire que l'on place à Avignon en raison du lieu de découverte de l'une des bases votives à son nom mais qui se trouvait peut-être sur la rive droite du Rhône, éventuellement sur le Mont Andaon (Christol et al. 2005-2006, 416-418).

Les inscriptions démontrent que la vitalité religieuse se maintient à l'Ermitage jusqu'au $\mathrm{II}^{\mathrm{e}} \mathrm{s}$. ap. J.-C., sinon jusqu'au III's. Cette dynamique s'était accompagnée d'une latinisation plus ou moins poussée des dénominations: substitution interprétative, associations interprétatives s'entremêlaient pour donner les dénominations des divinités. Mais le dieu qui, vraisemblablement, avait le rôle principal et fédérateur, l'hôte des lieux, était devenu Jupiter. Il avait rassemblé des dieux voisins, en même temps qu'il voyait venir à lui les populations des environs. Cette dynamique est une donnée remarquable qui se dégage bien de l'analyse des documents inscrits. Il est dommage qu'il soit pour l'instant impossible de présenter une traduction archéologique de données religieuses aussi suggestives.

M. ChrIstol - Université de Paris I-Panthéon-Sorbonne, UMR 8585.

J.-L. FICHES - Directeur de recherche CNRS, UMR 5140.

D. RABAY - Professeur certifié de lettres classiques, collège AnatoleFrance, 59 Sin Le Noble.

\section{NOTES}

1- Ce jour-là, J.-L. Fiches et D. Rabay étaient accompagnés de M. Gazenbeek et de L. Schneider que les auteurs tiennent à remercier chaleureusement pour les nombreuses observations qu'ils ont faites sur le terrain et que cet article met à profit. Les trois auteurs avaient auparavant effectué une visite sur le site et à la mairie de Collias en août 2004.

2- Vérifiée en 2005, alors qu' autrefois des monnaies romaines auraient été découvertes (Espérandieu 1934, 20).

3- Tous les blocs inscrits pour lesquels on connaît les circonstances de la découverte ont été trouvés en remploi dans l'église. Ils ont d'ailleurs été retaillés à cet effet. Ainsi, ceux qui furent cédés par la municipalité de Collias au Musée archéologique de Nîmes en 1887 provenaient, pour quatre d'entre eux (CIL, XII, 2973, 2971, 2975, 2979), de l'ancien autel et, pour deux autres, du mur méridional extérieur (CIL, XII, 5887, 2972), côté est, alors que la dédicace à Minerve (CIL, XII, 2974), conservée à la mairie de Collias, servait de linteau à la porte ouest, ouverte en 1697 (Durand, 1992, 135 et n. 12).

4- Sur le décor de deux d'entre eux et leur datation stylistique, Sauron 1983, catalogue ${ }^{\circ}$ VI-07 = CIL, XII, 2975 (Hadrien - Antonin le Pieux) et $n^{\circ}$ XIII.04 = CIL, XII, 2976 (fin du II ${ }^{\mathrm{e}} \mathrm{s}$.).

5- Contre Lejeune 1985 et Lambert 1997, 46, cette hypothèse a été défendue, en dernier lieu, par Sauze 2001, 13-14. Sur le Mont Andaon comme lieu de culte antique, voir Carru 2001; Christol et al. 2005.

6- C'est ainsi que se présente déjà l'interprétation d'Allmer 1881, 189, $\mathrm{n}^{\circ} 217$ : «Embellissements à un petit temple consacré au dieu local Aramon ».
7- L'un d'entre eux réside dans la singularité du terme qui, selon les dépouillements de Kajanto 1965, 281, ne semble pas connu ailleurs; voir aussi ibid., 127.

8- Sur le suffixe -illus/-illa, en latin, voir Kajanto 1965, 126-127 et 168170 , qui relève la fréquence de son apparition dans les provinces celtiques, et qui fait le rapprochement avec le suffixe celtique -illo-.

9- Lambert 1994, 33. Quelques exemples dans l'index de Lejeune 1985, 435-439: Escengil(l)os, à Villelaure dans le Vaucluse, selon G- 154 (ibid., 210); Ouimilla ou Ouimpilla à Ventabren, dans les Bouches-du-Rhône, selon G- 107 (ibid., 122) ; Ouirillios/Virilius à Genouilly, dans le Cher, selon G- 225 A (ibid., 334). Mais, bien sûr, les exemples se multiplient avec le développement de l'épigraphie latine. Il suffit d'en rester aux inscriptions de Gaule méridionale qui sont significatives: Divecillus, attesté à Cessero (Canava Divecilli f[ilia]: AE, 1968-1969, 383), mais aussi Excingilla (CIL, XII, 5004), Vassillus (CIL, XII, 2286), Vercillus/Vercilla (CIL, XII, 652, 724, 5085).

10- Il entre dans le groupe de termes, à valeur morale, qui définissent un caractère agréable (Kajanto, 1965, 73, 134).

11- $A E, 1978,463$ (inscription qui se trouve au Musée de Pont-SaintEsprit, mais qui provient des proches environs de Nîmes: $C A G$, $30-1,500$, notice 672 , inscr. 3 ).

12- CIL, XII, $3143=5902(H G L, X V, 2102=191 ;$ IAN, $143=582)$, voir $C A G, 30-1,348$, notice 311 , inscr. 61 .

13- Dans la présentation du texte par Allmer, lorsque celui-ci qualifie Aramon de «dieu local», il emploie vraisemblablement une formule générique, qui ne signifie pas que le dieu est le genius loci, mais un 
dieu au rayonnement limité du point de vue géographique. Ces explications relatives au rapport étroit qui devait exister entre la divinité Aramo et le lieu-dit Aramon reviennent dans les commentaires qui sont apportés à l'édition de l'inscription, dans $H G L, \mathrm{XV}$, 1510 et IAN, 548 (voir aussi Espérandieu 1934, 4, ainsi que Louis $1941,163, \mathrm{n}^{\circ} 184$, et $\left.146, \mathrm{n}^{\circ} 127\right)$. Ce n'est qu'une lecture rapide de ces ouvrages qui a pu faire indiquer, dans la $C A G$, dans l'introduction de la brève notice sur cette inscription, que le dieu Aramo serait le Génie du lieu même de la découverte. Ce même point de vue a été avancé par P. Finocchi 1994, 31-32.

14- Pour les Trois Gaules, W. Van Andringa (2002, 109 et n. 76) cite neuf attestations épigraphiques, généralement en contexte urbain, mais il note que la documentation archéologique signale des portiques dans nombre de sanctuaires ruraux.

15- On rappellera, en la matière, l'importance que revêtent, pour un sanctuaire rural ou pour un sanctuaire d'agglomération secondaire, les inscriptions de Moux et de Bram, dans la partie occidentale de la Narbonnaise. Pour l'inscription de Moux (CIL, XII, 5370, d'où ILS, 5421; HGL, XV, 148, dans la cité de Narbonne), qui fait connaître la construction des cellae sur l'emplacement du sanctuaire du dieu Larraso, voir Christol 2000. L'intérêt de ce texte a été méconnu par Fauduet, 1993, 31, n 403 (il y a confusion entre ce sanctuaire, bien attesté par l'épigraphie et par diverses trouvailles archéologiques, et un site archéologique considéré comme sanctuaire, qui se trouve à Douzens, dans le même canton de Capendu, mais à quelques kilomètres de Moux). Le site de Douzens est mentionné par Grenier 1959, 178$179, \mathrm{n}^{\circ} 104$, et le sanctuaire de Larraso est présenté à la p. 178, nº 103.

16- Et non sept, comme l'indique O. Hirschfeld, qui se fonde sur le dessin erroné reproduit par E. Germer-Durand (Germer-Durand 1875, 266).

17- Germer-Durand 1875, 265-266. Il considère que ces Corioledenses seraient les habitants de Collias, tandis que les Budenicenses seraient ceux de Bezuc, actuellement hameau sur la route entre Uzès et Alès, dans la commune de Baron (Germer-Durand 1868, 26). Le rapprochement est justifié par l'existence d'un site archéologique important, l'oppidum des Claparèdes: voir aussi, avec les mêmes conclusions, Louis, $1941,173, \mathrm{n}^{\circ} 212$. Mais on se reportera en définitive à Pène 2002, qui exclut ce rapprochement. On aurait pu, tout autant envisager un rapprochement avec Bezouce, bien plus proche de Collias: c'est un village voisin de Lédenon, sur la retombée des garrigues nîmoises dans la plaine du Vistre (Germer-Durand $1868,26)$.

18- Allmer 1880, 132-133, $\mathrm{n}^{\circ} 157$; remarques reprises par Otto Hirschfeld. On ne modifiait pas toutefois les identifications, comme le montrent les commentaires de HGL, XV, 452 et de IAN, 214.

19- On sera plus particulièrement attentif aux dénominations qui les font apparaître dans le contexte de la dénomination pérégrine: inventaires utiles, mais qu'il convient de compléter, par Chastagnol 1990.

20- $C I L$, XII, p. 926 et 927. Otto Hirschfeld allait contre l'avis de ses prédécesseurs qui avaient conservé la lecture de G. Guiran: notamment Ménard 1875, VII, 535, n 48, ainsi que Herzog 1864, 49, nº 243. Alors on connaissait bien les Suleviae, divinités apparaissant en groupe: elles appartiennent à la catégorie des Mères, fréquemment attestées en Gaule et dans les Germanies et transportées à Rome même par les soldats issus des provinces germaniques. Les auteurs de $H G L, \mathrm{XV}$, connaissent aussi ces divinités, mais n'osent pas corriger la copie de G. Guiran. La notice de Louis 1941, 163, n 184 , inscr. 4, est maladroite: «Déd. à Minerve, qui fait mention d'une Idennica».

21- C'était le point de vue de E. Germer-Durand 1875, 264. On le retrouve à peine modifié dans le commentaire de $H G L, \mathrm{XV}, 1512$ :
«La Sulève de l'Eyssene aurait été la personnification, non pas du ruisseau de l'Eyssène, car elle aurait dû alors s'appeler Sulivia Idenna, mais de la forêt qui, à l'époque du vœu dont il s'agit, entourait la source de ce ruisseau ». Otto Hirschfeld puis H.Dessau avaient signalé cette hypothèse dans leurs brefs commentaires. Cette petite rivière est un affluent de l'Alzon, qui à son tour se jette dans le Gardon à hauteur de Collias. On retrouve ce point de vue chez P. Finocchi 1994, 67-68, cf. 97.

22- Ce document, connu dès le XVII' siècle, passa par la suite dans la collection de Séguier (Darde, Christol 2003, 34, n³).

23- H. conservée $50 \mathrm{~cm}, 1$ conservée $40 \mathrm{~cm}$, ép. $37 \mathrm{~cm}$. Dimensions du dé: h. conservée $39,5 \mathrm{~cm}, 1.34,5 \mathrm{~cm}$, ép. $29 \mathrm{~cm}$.

24- Cette inscription n'est pas inventoriée dans Louis 1941, 162, n 180. En revanche, voir $C A G, 30-3,736$, notice $346, \mathrm{n}^{\circ} 19$, inscr. 1.

25- On ne trouve rien à ce sujet dans l'article pourtant important de Chastagnol 1999.

26- À la ligne 6, il faut restituer l'emplacement du praenomen, mais comme le remarque O. Hirsfeld, A. Allmer a peut-être abusivement lu la lettre L. À la dernière ligne on a signalé que le $\mathrm{O}$, de plus grandes dimensions (les lettres ont une hauteur de $4 \mathrm{~cm}$ à cette ligne, le $\mathrm{O}$ a $5 \mathrm{~cm}$ de diamètre) prenait la forme d'une rouelle. Déjà A. Allmer l'avait remarqué $\left(1880,135, \mathrm{n}^{\circ} 162\right)$ une lettre en partie effacée après CESTI, à la dernière ligne, paraît être un $\mathrm{O}$ dans lequel on aurait tracé une rosace ou la rouelle fulgurante de Jupiter. Otto Hirschfeld lui-même, qui a pu voir l'autel lors de son ultime voyage en 1886, le confirme: «7 ex. rotae pars sinistra superest»). La lettre est incomplète. Les anciennes copies font apparaître même une lettre F qui viendrait soit après le mot CESTIO soit seulement après les lettres CESTI. À la page 894, l'index de CIL, XII comprend la dénomination de la personne qui consacre l'autel, sous la forme Lucilius Cestif., c'est-à-dire sous la forme d'une dénomination pérégrine.

27- Autel à la roue, entre deux foudres. Mais dédié à Jupiter Optimus Maximus: l'assimilation au dieu romain est ici totale.

28- Autel à la roue, dédié Iovi et Augusto, de date assez haute ( $\left(^{\mathrm{er}} \mathrm{s} . \mathrm{ap}\right.$. J.-C.). Localisation erronée dans la commune de Vers, par Louis $1941,163, n^{\circ} 182$.

29- Lecture corrigée et transmise à $C A G, 30-3$. Voir aussi Buffat et al. 2002, 222-223. L'inscription se trouve actuellement au Musée d'Avignon.

30- Il faut aussi tenir compte, pour cette association de Jupiter et de la Terre Mère, du petit autel, provenant de Nîmes (CIL, XII, 3071; $H G L, \mathrm{XV}, 4$; CAG, 30-1, 502, notice 681, inscr. 2 et CAG, 30-2, 324 , notice $082, n^{\circ} 2$ ), qui faisait partie de la collection de Séguier et qui se trouve actuellement au Musée des Antiquités Nationales, à Saint-Germain-en-Laye (Darde, Christol 2003, 33, n²).

31- On ne peut intégrer au dossier le témoignage qui provient du sanctuaire de la Fontaine, car c'est Jupiter Héliopolitain qui est associé à Nemausus (CIL, XII, 3072; HGL, XV, $2=461$; mais aussi CIL, XII, 3070 et add.; HGL, XV, 3 = 198 ; IAN, 3 = 94).

32- Néanmoins, pour Mars, le phénomène d'effacement de la diversité religieuse par le nom romain est un phénomène marquant. Voir les remarques de Christol 2003a, 411-423, notamment 420-422.

33- Sur le caractère local de la divinité et les rapports significatifs entre son culte et la population, Christol 2003b, 49-56; dans la cité de Nîmes, Bermond et al. 1998, 119-154. Paola Finocchi (1994, 42-43) met en évidence son caractère guerrier, qui confirme l'assimilation avec Mars.

34- Duval 1993, il s'agirait d'une déesse de la fécondité. 


\section{BIBLIOGRAPHIE}

\section{Abréviations}

CAG: Carte Archéologique de la Gaule. Le Gard, 30-2, 330, notice 085, n ${ }^{\circ}{ }^{*}$ par Michel Provost et al.

HGL XV : Barry Ed., Germer-Durand E. 1892 - Recueil des Inscriptions Antiques de la province de Languedoc, Toulouse, Privat (Histoire générale de Languedoc, XV), 1892, XV-1251 p.

IAN : Germer-Durand E. et Fr., Allmer A., Inscriptions antiques de Nîmes, Toulouse, Privat, 1893, 1100 p.

ILGN : Espérandieu É., Inscriptions latines de Gaule Narbonnaise, Paris, E. Leroux, (suppl. au C.I.L., XII) 1929, II-224 p.

ILN: Inscriptions Latines de Narbonnaise. Paris, CNRS Editions, 1985-

ILS : H. Dessau, Inscriptiones Latinae Selectae. Berlin, 1892-1916 (rééd. 1954-55; 1962).

\section{Références dans le texte}

Allmer 1880 : ALLMER (A.) - Rev. épigr., I (9), 1880, p. 132-135.

Allmer 1881 : ALLMER (A.) - Rev. épigr., I (12), 1881, p. 189.

Arcelin 1979: ARCELIN (P.) - La nécropole préromaine de l'Arcoule, commune du Paradou (Bouches-du-Rhône). DocAMérid, 2, 1979, p. 133-156.

Arcelin et al. 1992: ARCELIN (P.), DEDET (B.) et SCHWALLER (M.) Espaces publics, espaces religieux protohistoriques en Gaule méridionale. DocAMérid., 15, 1992, p.181-242.

Arcelin, Gruat 2003 : ARCELIN (P.), GRUAT (Ph.) et collab. La France du Sud-Est (Languedoc-Roussillon, Midi-Pyrénées, Provence-Alpes-Côte-d'Azur). In : ARCELIN (P.), BRUNAUX (J.-L.) dir. - Cultes et sanctuaires en France à l'âge du Fer, Gallia, 60, 2003, p. 169-241.

Bauquier 1946: BAUQUIER (H.) - Inscription nouvelle au Musée lapidaire, Le Vieux Nîmes, Bulletin de la Commission Municipale d'Archéologie, 21, 1946, 2.

Bayol 1980: abbé BAYOL (J.) - Ermitage de Collias, Notre-Dame de Laval, Gorges du Gardon. Collias, Aux Amis de l'Ermitage, 1980, 29 p. (année du Patrimoine).

Bermond et al. 1998: BERMOND (I.) et BRIAND (A.) collab., CHRISTOL (M.) collab., STERNBERG (M.) collab. - Le sanctuaire gallo-romain de Mars à Balaruc-les-Bains. RANarb, 31, 1998, p. $119-154$

Blétry-Sébé 1986: BLÉTRY-SÉBÉ (S.) - Le culte rural des eaux dans les cités antiques de Nîmes, Béziers et Lodève. In: Études Languedociennes. Actes du $110^{e}$ Congrès Nat. Soc. Savantes, Montpellier, 1985. Paris, CTHS, 1986, 241-253.

Buffat et al. 2002 : BUFFAT (L.), CHRISTOL (M.), PÉLAQUIER (É.) et PETITOT $(\mathrm{H}$.$) - Le problème d'interprétation des établissements$ ruraux de grande dimension: quelques cas en Languedoc. RANarb, 35, 2002, 199-239.

Carru 2001 : CARRU (D.) - Aux origines du mont Andaon. Villeneuve-lès-Avignon et sa proche région jusqu'au Moyen Âge. In: BARRUOL (G.), BACOU (R.) et GIRARD (A.) - L'abbaye Saint-André de Villeneuve-lès-Avignon: histoire, archéologie, rayonnement. Mane, Les Alpes de Lumière, 2001, 15-22 (Les Cahiers de Salagon, 4).
Chastagnol 1990: CHASTAGNOL (A.) - L'onomastique de type pérégrin dans les cités de la Gaule Narbonnaise. MEFRA, 102 1990, 573-593 (= La Gaule romaine et le droit latin. Lyon, 1995, 51-71).

Chastagnol 1999: CHASTAGNOL (A.) - Réflexions sur l'évolution du formulaire épigraphique dans les dédicaces locales du culte impérial. RANarb, 32, 1999, p. 5-9.

Christol 1990 : CHRISTOL (M.) - Les découvertes épigraphiques, 4, Autel à Mars Melovius. In DARDE (D.) com. - Archéologie à Nîmes. Bilan de 40 années de recherches et découvertes (19501990), ville de Nîmes, Musée archéologique, juin 1990, Nîmes, p. 175-207.

Christol 2000 : CHRISTOL (M.) - Un pagus dans l'arrière-pays de Narbonne (CIL, XII, 5390). In: Epigraphai. Miscellanea in onore di Lidio Gasperini (a cura di Gianfranco Paci). Tivoli, I, 2000, p. 247-273.

Christol 2003a: CHRISTOL (M.) - L'épigraphie et les dieux du Plateau des Poètes à Béziers. RANarb, 36, 2003, p. 411-423.

Christol 2003b : CHRISTOL (M.) - À propos d'une inscription de Lattes relative à Deus Mars Augustus: l'acte religieux et le don. Archéologie en Languedoc, 27, 2003, p. 49-56.

Christol et al. 1987: CHRISTOL (M.), GASCOU (J.) et JANON (M.) Les seviralia ornamenta gratuita dans une inscription de Nîmes. Latomus, 46, 1987, p. 388-398.

Christol et al. 2005-2006 : CHRISTOL (M.), FICHES (J.-L.), GASCO (Y.) et MICHELOZZI (A.) - Une nouvelle dédicace de T(itus) Carisius, praetor Volcarum, près d'Ugernum (Beaucaire, Gard). RANarb, 38-39, 2005-2006, 409-423.

Christol, Deneux 2001 : CHRISTOL (M.) et DENEUX (C.) - La latinisation de l'anthroponymie dans la cité de Nîmes à l'époque impériale (début de la seconde moitié du Irr siècle av. J.-C. III ${ }^{\mathrm{e}}$ siècle ap. J.-C.): les données de la dénomination pérégrine. In: DONDIN-PAYRE (M.) et RAEPSAET-CHARLIER (M.-Th.) éd. Noms, identités culturelles et romanisation sous le Haut-Empire romain. Bruxelles, 2001, p.39-54.

Darde, Christol 2003: DARDE (D.) et CHRISTOL (M.) - La collection Séguier au Musée Archéologique de Nîmes. Nîmes, 2003, 95 p. (Cahier des musées et monuments $n^{\circ} 12$ ).

Delamarre 2001: DELAMARRE (X.) - Dictionnaire de la langue gauloise. Paris, 2001.

Durand 1992: DURAND (G.) - L'Ermitage Notre-Dame de Laval à Collias (Gard). In: Travaux offerts à Marcel Durliat. Toulouse, 1992, p. 133-143.

Duval 1993 : DUVAL (P.-M.) - Les dieux de la Gaule. Paris, Payot, 1993.

Espérandieu 1934 : ESPÉRANDIEU (É.) - Répertoire Archéologique du département du Gard. Période gallo-romaine. Montpellier, FHLMR, 1934, 82 p.

Espérandieu 1907-1938: ESPÉRANDIEU (É.) - Recueil général des bas-reliefs, statues et bustes de la Gaule romaine. Paris, 1907-1938, 11 vol.

Fabre 1980: FABRE (G.) - Les karsts du Languedoc oriental: recherches hydrogéomorphologiques. Nîmes, Association française de karstologie (Mémoire n 2), 1980, 446 p., 23 pl. 
Fabre, Pey 1997: FABRE (G.) et PEY (J.) - Le Gardon et ses gorges. Montpellier, Les Presses du Languedoc, 1997, 159p.

Fauduet 1993: FAUDUET (I.) - Atlas des sanctuaires romano-celtiques de Gaule. Les fanums. Paris, Errance, 1993.

Finocchi 1994 : FINOCCHI (P.) - Dizionario delle divinità della Gallia Narbonense. Rome, 1994.

Fugier 1963: FUGIER (H.) - Recherches sur l'expression du sacré dans la langue latine. Paris, 1963.

Genty et al. 2002 : GENTY (P.-Y.), FICHES (J.-L.) et CHRISTOL (M.) collab., PÈNE (J.-M.) collab. - Aramo. In : FICHES (J.-L.) dir. - Les agglomérations gallo-romaines en LanguedocRoussillon. Lattes, ADAL, 2002, p. $730-736$ (Monographies d'Archéologie Méditerranéenne, 14).

Germer-Durand 1868: GERMER-DURAND (E.) - Dictionnaire topographique du département du Gard. Paris, 1868.

Germer-Durand 1875 : GERMER-DURAND (E.) - Découvertes archéologiques faites à Nîmes et dans le Gard, pendant l'année 1873. Mémoires de l'Académie du Gard, 1875 [1876], p. 268-269.

Guillet et al. 1992: GUILLET (É.), LELIÈVRE (V.), PAILLET (J.-L.), PISKORZ (M.), RECOLIN (A.) et SOUQ (Fr.) - Un monument à portique tardo-hellénistique près de la source de la Fontaine, à Nîmes (Gard). DocAMérid., 15, 1992, p. 57-89.

Grenier 1959: GRENIER (A.) - Carte archéologique de la Gaule romaine, XII. Aude. Paris, PUF, 1959.

Herzog 1864 : HERZOG (E.) - Galliae Narbonensis provinciae romanae historia, descriptio, institutorum expositio, Appendix epigraphica. Leipzig, 1864.

Kajanto 1965 : KAJANTO (I.) - The Latin cognomina. Helsinki, 1965.

Lambert 1994: LAMBERT (P.-Y.) - La langue gauloise. Paris, 1994.

Lambert 1997 : LAMBERT (P.-Y.) - L'épigraphie gallo-grecque. In : CHRISTOL (M.), MASSON (O.) éd. - Actes du Xe Congrès international d'épigraphie grecque et latine (Nîmes, 4-9 octobre 1992). Paris, Publications de la Sorbonne, 1997, p. 35-50.

Lejeune 1985: LEJEUNE (M.) - Recueil des Inscriptions gauloises, I. Les inscriptions gallo-grecques. Paris, 1985.

Louis 1941: LOUIS (M.) - Carte archéologique de la Gaule romaine (département du Gard). Paris, PUF, 1941.

Marcelin 1926: MARCELIN (P.) - Contribution à l'étude géographique de la garrigue nîmoise. DES, géographie, Institut de géographie, Lyon, Études rhodaniennes, 1926, 174 p.
Marrou 1932: MARROU (H.-I.) - Le culte des sources dans la cité gallo-romaine de Nîmes. In: Actes du Congrès de l'Association G. Budé, Nîmes, 1932. Paris, 1932, p. 186-195.

Mazauric 1905: MAZAURIC (F.) - Stations du Mas de Laval. Bull. de la Soc. d'Étude des Sciences Naturelles de Nîmes, 33, 1905, p. XXX.

Ménard 1875: MÉNARD (L.) - Histoire civile, ecclésiastique et littéraire de la ville de Nîmes. Nîmes, 1875 (2ééd.).

Paillet 2000: PAILLET (J.-L.) - La deuxième vie de l'aqueduc ou le remploi de ses concrétions dans les monuments médiévaux. In: FABRE (G.), FICHES (J.-L.), PAILLET (J.-L.) dir. - L'aqueduc antique de Nîmes et le Pont du Gard. Archéologie, géosystème et histoire. Paris, CNRS Éditions, 2000, $2^{\mathrm{e}}$ éd. revue et augmentée, p. 423-441 (CRA Monographies, hors série).

Pène 2002: PÈNE (J.-M.) - Les Claparèdes et l'oppidum de la Dame de Bruyès, Aigaliers et Baron (Gard). In : FICHES (J.-L.) dir. Les agglomérations gallo-romaines en Languedoc-Roussillon. Lattes, ADAL, 2002, 829-844 (Monographies d'Archéologie Méditerranéenne, 14).

Petry 1982: PETRY (F.) - Nouvelles divinités du Strasbourg romain. Cahiers alsaciens d'archéologie, d'art et d'histoire, 1982, p. 5760.

Provost et al. 1999: PROVOST (M.) et al. - Le Gard. Paris, Académie des Inscriptions et Belles-Lettres, 1999, 2 vol. 865 p., (Carte Archéologique de la Gaule, 30/2-3).

Roth Congès 1992 : ROTH CONGÈS (A.) - Monuments publics d'époque tardo-hellénistique à Glanon (B.-du-Rh.). DocAMérid, 15, 1992, p. 50-56.

Sauron 1983: SAURON (G.) - Les cippes funéraires gallo-romains à décor de rinceaux de Nîmes et de sa région. Gallia, 41, 1983, p. 59-110.

Sauvage 1992 : SAUVAGE (L.) - Le sanctuaire protohistorique de la Fontaine à Nîmes, à la lumière des découvertes récentes. DocAMérid, 15, 1992, p. 112-116.

Sauze 2001: Sauze (É.) - Le toponyme Andaon. In: BARRUOL (G.), BACOU (R.) et GIRARD (A.) - L'abbaye Saint-André de Villeneuve-lès-Avignon: histoire, archéologie, rayonnement. Mane, Les Alpes de Lumière (Les Cahiers de Salagon, 4), 2001, p. 11-14.

Van Andringa 2002 : VAN ANDRINGA (W.) - La religion en Gaule romaine. Piété et politique (Irr-IIIe siècle apr. J.-C.). Paris, Errance, 2002, 336p. 